\title{
Sloan Digital Sky Survey Imaging of Low Galactic Latitude Fields: Technical Summary and Data Release
}

\section{Citation}

Finkbeiner, Douglas P., Nikhil Padmanabhan, David J. Schlegel, Michael A. Carr, James E. Gunn, Constance M. Rockosi, Maki Sekiguchi, et al. 2004. "Sloan Digital Sky Survey Imaging of Low Galactic Latitude Fields: Technical Summary and Data Release." The Astronomical Journal 128 (5) (November): 2577-2592. doi:10.1086/425050.

\section{Published Version}

doi:10.1086/425050

\section{Permanent link}

http://nrs.harvard.edu/urn-3:HUL.InstRepos:33370049

\section{Terms of Use}

This article was downloaded from Harvard University's DASH repository, and is made available under the terms and conditions applicable to Other Posted Material, as set forth at http:// nrs.harvard.edu/urn-3:HUL.InstRepos:dash.current.terms-of-use\#LAA

\section{Share Your Story}

The Harvard community has made this article openly available.

Please share how this access benefits you. Submit a story. 


\title{
SLOAN DIGITAL SKY SURVEY IMAGING OF LOW GALACTIC LATITUDE FIELDS: TECHNICAL SUMMARY AND DATA RELEASE
}

Douglas P. Finkbeiner, ${ }^{1,2}$ Nikhil Padmanabhan, ${ }^{3}$ David J. Schlegel, ${ }^{2}$ Michael A. Carr, ${ }^{2}$ James E. Gunn, ${ }^{2}$ Constance M. Rockosi,,${ }^{1,4}$ Maki Sekiguchi, ${ }^{5}$ Robert H. Lupton, ${ }^{2}$ G. R. Knapp, ${ }^{2}$ Željko Ivezić,,${ }^{2}$ Michael R. Blanton, ${ }^{6}$ David W. Hogg, ${ }^{6}$ Jennifer K. Adelman-McCarthy, ${ }^{7}$ James Annis, ${ }^{7}$ Jeffrey Hayes, ${ }^{8}$ Ellynne Kinney, ${ }^{9}$ Daniel C. Long, ${ }^{9}$ Uroš Seljak, ${ }^{3}$ Michael A. Strauss, ${ }^{2}$ Brian Yanny, ${ }^{7}$ Marcel A. Agüeros, ${ }^{4}$ Sahar S. Allam, ${ }^{10}$ Scott F. Anderson, ${ }^{4}$ Neta A. Bahcall, ${ }^{2}$ Ivan K. Baldry,${ }^{11}$ Mariangela Bernardi, ${ }^{12}$ William N. Boroski,${ }^{7}$ John W. Briggs, ${ }^{13}$ J. Brinkmann, ${ }^{9}$ Robert J. Brunner, ${ }^{14}$ Tamás Budavári ${ }^{11}$ Francisco J. Castander, ${ }^{15}$ Kevin R. Covey,${ }^{4}$ István Csabai, ${ }^{11}, 16$ Mamoru Doi, ${ }^{17}$ Feng Dong, ${ }^{2}$ Daniel J. Eisenstein ${ }^{18}$ Xiaohui Fan, ${ }^{18}$ Scott D. Friedman, ${ }^{19}$ Masataka Fukugita, ${ }^{20}$ Bruce Gillespie, ${ }^{9}$ Eva K. Grebel, ${ }^{21}$ Vijay K. Gurbani, ${ }^{7}, 22$ Ernst de Haas, ${ }^{2}$ Frederick H. Harris, ${ }^{23}$ John S. Hendry, ${ }^{7}$ Gregory S. Hennessy ${ }^{24}$ Sebastian Jester, ${ }^{7}$ David E. Johnston, ${ }^{2}$ Anders M. Jorgensen, ${ }^{25}$ Mario Jurić, ${ }^{2}$ Stephen M. Kent, ${ }^{7}$ Alexei Yu. Kniazev, ${ }^{21}$ Jurek Krzesiński, ${ }^{9,26}$ R. French Leger, ${ }^{7}$ Huan Lin, ${ }^{7}$ Jon Loveday, ${ }^{27}$ Ed Mannery, ${ }^{4}$ David Martínez-Delgado, ${ }^{21}$ Peregrine M. McGehee, ${ }^{10,28}$ Avery Meiksin, ${ }^{29}$ Jefrrey A. Munn, ${ }^{23}$ Eric H. Neilsen, Jr., ${ }^{7}$ Peter R. Newman, ${ }^{9}$ Atsuko Nitta, ${ }^{9}$ George Pauls, ${ }^{2}$ Thomas R. Quinn, ${ }^{4}$ R. R. Rafikov, ${ }^{30}$ Gordon T. Richards, ${ }^{2}$ Michael W. Richmond, ${ }^{31}$ Donald P. Schneider, ${ }^{32}$ Joshua Schroeder, ${ }^{2}$ Kazu Shimasaku, ${ }^{33}$ Walter A. Siegmund, ${ }^{34}$ J. Allyn Smith, ${ }^{25,35}$ Stephanie A. Snedden, ${ }^{9}$ Albert Stebbins, ${ }^{7}$ Alexander S. Szalay, ${ }^{11}$ Gyula P. Szokoly, ${ }^{36}$ Max Tegmark, ${ }^{37}$ Douglas L. Tucker, ${ }^{7}$ Alan Uomoto, ${ }^{11,38}$ Daniel E. Vanden Berk, ${ }^{39}$ David H. Weinberg, ${ }^{40}$ Andrew A. West, ${ }^{4}$ Naoki Yasuda, ${ }^{20}$ D. R. Yocum, ${ }^{7}$ Donald G. York, ${ }^{41,42}$ and Idit Zehavi ${ }^{18}$ Received 2004 May 10; accepted 2004 August 12

\begin{abstract}
The Sloan Digital Sky Survey (SDSS) mosaic camera and telescope have obtained five-band optical-wavelength imaging near the Galactic plane outside of the nominal survey boundaries. These additional data were obtained during commissioning and subsequent testing of the SDSS observing system, and they provide unique wide-area imaging data in regions of high obscuration and star formation, including numerous young stellar objects, HerbigHaro objects, and young star clusters. Because these data are outside the survey regions in the Galactic caps, they are not part of the standard SDSS data releases. This paper presents imaging data for 832 square degrees of sky (including repeats), in the star-forming regions of Orion, Taurus, and Cygnus. About $470 \mathrm{deg}^{2}$ are now released to the public, with the remainder to follow at the time of SDSS Data Release 4. The public data in Orion include the star-forming region NGC 2068/NGC 2071/HH 24 and a large part of Barnard's loop.
\end{abstract}

Key words: atlases — catalogs — surveys

\footnotetext{
Hubble Fellow.

2 Department of Astrophysical Sciences, Peyton Hall, Princeton University, Princeton, NJ 08544.

3 Joseph Henry Laboratories, Department of Physics, Princeton University, Princeton, NJ 08544.

${ }_{5}^{4}$ Department of Astronomy, University of Washington, Box 351580, Seattle, WA 98195.

5 Japan Participation Group, c/o Institute for Cosmic Ray Research, University of Tokyo, 5-1-5 Kashiwa, Kashiwa City, Chiba 277-8582, Japan.

${ }^{6}$ Center for Cosmology and Particle Physics, Department of Physics, New York University, 4 Washington Place, New York, NY 10003.

${ }^{7}$ Fermi National Accelerator Laboratory, P.O. Box 500, Batavia, IL 60510.

8 Department of Physics, Catholic University of America, Washington, DC 20064.

9 Apache Point Observatory, P.O. Box 59, Sunspot, NM 88349.

${ }^{10}$ Department of Astronomy, New Mexico State University, 1320 Frenger Mall, Las Cruces, NM 88003-8001.

11 Center for Astrophysical Sciences, Department of Physics and Astronomy, 3400 North Charles Street, Johns Hopkins University, Baltimore, MD 21218.

12 Department of Physics, Carnegie Mellon University, 5000 Forbes Avenue, Pittsburgh, PA 15213.

${ }^{13}$ Yerkes Observatory, University of Chicago, 373 West Geneva Street, Williams Bay, WI 53191.

${ }^{14}$ Department of Astronomy, University of Illinois, 1002 West Green Street, Urbana, IL 61801.

15 Institut d'Estudis Espacials de Catalunya, CSIC, Gran Capita 2-4, E-08034 Barcelona, Spain.

${ }_{17}^{16}$ Department of Physics of Complex Systems, Eötvös Loránd University, Pf. 32, H-1518 Budapest, Hungary.

17 Institute of Astronomy, University of Tokyo, 2-21-1 Osawa, Mitaka, Tokyo 181-0015, Japan.

${ }^{18}$ Steward Observatory, 933 North Cherry Avenue, Tucson, AZ 85721.

19 Space Telescope Science Institute, 3700 San Martin Drive, Baltimore, MD 21218.

20 Institute for Cosmic Ray Research, University of Tokyo, 5-1-5 Kashiwa, Kashiwa City, Chiba 277-8582, Japan.

${ }^{21}$ Max-Planck Institut für Astronomie, Königstuhl 17, D-69117 Heidelberg, Germany.

${ }^{22}$ Lucent Technologies, 2000 Lucent Lane, Naperville, IL 60566.

${ }^{23}$ US Naval Observatory, Flagstaff Station, P.O. Box 1149, Flagstaff, AZ 86002.

${ }^{24}$ US Naval Observatory, 3540 Massachusetts Avenue, NW, Washington, DC 20392.

${ }^{25}$ Space Instrumentation and System Engineering Group, Mail Stop D448, Los Alamos National Laboratory, Los Alamos, NM 87545.

26 Obserwatorium Astronomiczne na Suhorze, Akademia Pedagogiczna w Krakowie, ulica Podchorążych 2, PL-30-084 Kraców, Poland.

27 Astronomy Centre, University of Sussex, Falmer, Brighton BN1 9QJ, England.

${ }^{28}$ Global Controls Group, Mail Stop H820, Los Alamos National Laboratory, Los Alamos, NM 87545.

${ }^{29}$ Institute for Astronomy, Royal Observatory, Blackford Hill, Edinburgh EH9 3HJ, Scotland.

${ }^{30}$ Institute for Advanced Study, Einstein Drive, Princeton, NJ 08540.

31 Department of Physics, Rochester Institute of Technology, 84 Lomb Memorial Drive, Rochester, NY 14623-5603.

32 Department of Astronomy and Astrophysics, 525 Davey Laboratory, Pennsylvania State University, University Park, PA 16802.
} 


\section{INTRODUCTION}

The Sloan Digital Sky Survey (SDSS) is a five-band photometric survey of 8500 square degrees of the northern sky and a concurrent redshift survey of nearly a million galaxies and 100,000 quasars selected from the imaging survey (York et al. 2000). The primary purpose of the project is to investigate the large-scale structure of the universe and pursue other extragalactic science. The official survey region therefore largely lies above Galactic latitude $|b|>30^{\circ}$ and was carefully chosen to minimize the effect of the troublesome dust near the Galactic plane. This naturally excludes some of the most beautiful parts of the sky, and those areas of most interest for Galactic science.

However, a significant amount of imaging data has been obtained at low Galactic latitude by SDSS, both during telescope commissioning and for calibration at sidereal times when the main survey region was unavailable. During commissioning, the SDSS camera (Gunn et al. 1998) was operational before the telescope control system was stable. The camera data acquisition is carried out in drift-scan, or "time-delay and integrate" (TDI), mode, with the camera crossing the sky at the sidereal rate; the large field of view $\left(3^{\circ}\right)$ mandates that this be done along great circles. Accordingly, much of the commissioning work was done by parking the telescope at the celestial equator and drift-scanning the sky. During commissioning observations in the fall of 1998 (when the south Galactic polar cap was available for observation), the drift-scanning continued outside the SDSS survey area and passed through NGC 2068 and NGC 2071 in Orion. Many of the commissioning runs produced data of high scientific quality, some of which are part of the SDSS Early Data Release (EDR; Stoughton et al. 2002), where a detailed description of the imaging data can be found. SDSS runs 259 and 273 generated targets for spectroscopic commissioning, resulting in the first extremely metal-poor galaxy found by SDSS (Kniazev et al. 2003), but have never been made public. Since 1999, additional imaging data at low Galactic latitudes have been obtained for a variety of testing and calibration purposes. However, none of these low-latitude data, external to the SDSS survey area, are included in the SDSS data releases (to date, the EDR, the first data release, DR1 [Abazajian et al. 2003], and DR2 [Abazajian et al. 2004]). We have reduced and calibrated these data as part of a rereduction and recalibration of all the imaging data (the "übercalibration"; Schlegel et al. 2004), and we describe these data in the present paper.

The areas of sky observed are described in $\S 2$. The data products described herein are very similar to those distributed

\footnotetext{
${ }^{33}$ Department of Astronomy and Research Center for the Early Universe, School of Science, University of Tokyo, 7-3-1 Hongo, Bunkyo, Tokyo 1130033, Japan.

${ }^{34}$ Institute for Astronomy, University of Hawaii, 2680 Woodlawn Drive, Honolulu, HI 96822.

${ }^{35}$ Department of Physics and Astronomy, University of Wyoming, P.O. Box 3905, Laramie, WY 82071.

${ }^{36}$ Max-Planck-Institut für extraterrestrische Physik, Giessenbachstrasse 1, D-85741 Garching, Germany.

${ }^{37}$ Department of Physics and Astronomy, University of Pennsylvania, 209 South 33rd Street, Philadelphia, PA 19104.

${ }^{38}$ Carnegie Observatories, 813 Santa Barbara Street, Pasadena, CA 91101.

39 Department of Physics and Astronomy, University of Pittsburgh, 3941 O'Hara Street, Pittsburgh, PA 15260.

${ }_{40}$ Department of Astronomy, Ohio State University, 140 West 18th Avenue, Columbus, $\mathrm{OH} 43210$.

${ }^{41}$ Department of Astronomy and Astrophysics, University of Chicago, 5640 South Ellis Avenue, Chicago, IL 60637.

${ }^{42}$ Enrico Fermi Institute, University of Chicago, 5640 South Ellis Avenue, Chicago, IL 60637.
}

in the previous SDSS data releases but differ in photometric calibration and data format. These differences are described in $\S 3$, and some examples of science applications are briefly discussed in $\S 4$. The data from 1998-1999 are publicly released with this paper and may be accessed via the World Wide Web. ${ }^{43}$

\section{THE DATA}

\subsection{Sky Coverage}

The sky coverage of the imaging runs to date outside the SDSS area is given in Table 1 and Figure 1. The figure shows the footprint of the survey (including these runs) in equatorial coordinates and indicates the approximate locations of the images in Figures 2, 3, and 4. Table 1 provides information about the location and image quality of each run. An imaging run consists of six long images in each filter, each the width of one CCD (13.'52 on the sky) and separated by slightly less than one CCD width (11.'65), produced by the six columns of CCDs in the mosaic camera. A run may last the entire night and be over $100^{\circ}$ long, or it can be shorter. A strip is the area covered by the six camera columns from one survey pole to the other; a stripe is a pair of interleaving strips and completely covers a $2.5 \times 180^{\circ}$ stripe on the sky. The imaging data are divided into $8.98 \times 13.52$ frames for further processing; the aligned frames in the five bands are called a field. Since the sky passes through the $u, g, r, i$, and $z$ SDSS filters in succession (in order riuzg), there is a ramp-up time corresponding to 10 frames at the start of each imaging run. Focus and tracking adjustments are made at the beginning of each run, adding variable amounts of overhead to the ramp-up time. The range of fields for each run given in Table 1 defines the range of useful data. A detailed description of the observing procedures is given by Gunn et al. (1998) and York et al. (2000).

The imaging runs comprising the "Orion" data set, released in the present paper, were made in the fall of 1998 and fall of 1999. As Table 1 and Figure 1 show, there have been quite a few low-latitude observations since then, usually for system checking and calibration. Nonphotometric data are included, because proper-motion studies can make use of the astrometry even in unphotometric runs. The data listed in Table 1 are those which, after reduction and processing, prove to be of science quality, and the photometric reliability is indicated.

\subsection{Processing}

The SDSS photometric pipeline consists of four sequential steps: ssc and psp (point-spread function estimation), astrom (Pier et al. 2003; astrometry), and frames (object identification, deblending, and photometry; Lupton et al. 2001; Stoughton et al. 2002; Lupton et al. 2002, 2004). These pipelines run with little human intervention. The photometric pipeline corrects the imaging data by interpolating over defects such as bad columns and cosmic rays; provides flat-field, photometric, and astrometric calibration; and identifies, deblends, measures, and classifies objects. The resulting outputs consist of corrected frames, atlas image cutouts for every object, and a catalog of object positions, magnitudes, and image classifications in the five SDSS bands. The catalog outputs also include flags that describe the image processing, including whether the object contains any saturated pixels, whether bad data were interpolated over, and whether the object was deblended. Careful attention to these processing flags is critical

\footnotetext{
${ }^{43}$ At http://photo.astro.princeton.edu (hereafter "the Web site"). Future data releases will also be accessible at this site.
} 
TABLE 1

SDSS Galactic Plane Runs

\begin{tabular}{|c|c|c|c|c|c|c|c|c|c|c|c|c|}
\hline $\begin{array}{c}\text { Run } \\
\text { (1) }\end{array}$ & $\begin{array}{l}\text { UT Date } \\
\text { (2) }\end{array}$ & $\begin{array}{l}\text { MJD } \\
(3)\end{array}$ & $\begin{array}{l}\text { Strip } \\
\text { (4) }\end{array}$ & $\begin{array}{c}\text { Node } \\
\text { (deg) } \\
(5)\end{array}$ & $\begin{array}{l}\text { Incl. } \\
\text { (deg) } \\
\text { (6) }\end{array}$ & $\begin{array}{l}F_{0} \\
(7)\end{array}$ & $\begin{array}{l}F_{1} \\
(8)\end{array}$ & $\begin{array}{c}A \\
\left(\mathrm{deg}^{2}\right) \\
(9)\end{array}$ & $\begin{array}{c}l \\
(\mathrm{deg}) \\
(10)\end{array}$ & $\begin{array}{c}b \\
(\mathrm{deg}) \\
(11)\end{array}$ & $\begin{array}{c}\text { PSF } \\
(\operatorname{arcsec}) \\
(12)\end{array}$ & $\begin{array}{c}\text { Phot.? } \\
\text { (13) }\end{array}$ \\
\hline $211 \ldots \ldots \ldots \ldots . . .$. & 1998 Oct 29 & 51,115 & $82 \mathrm{~S}$ & 283.22 & 0.01 & 11 & 385 & 71.2 & 210.2 & -3.3 & 1.36 & $\mathrm{Y}$ \\
\hline $250 \ldots \ldots \ldots \ldots \ldots \ldots$ & 1998 Nov 16 & 51,133 & $82 \mathrm{~N}$ & 62.10 & 0.02 & 11 & 455 & 84.5 & 201.9 & -18.0 & 1.55 & $\mathrm{Y}$ \\
\hline $259 \ldots \ldots \ldots \ldots$ & 1998 Nov 17 & 51,134 & $82 \mathrm{~N}$ & 299.41 & 0.01 & 12 & 567 & 105.6 & 206.7 & -9.5 & 1.19 & $\mathrm{Y}$ \\
\hline $273 \ldots \ldots \ldots \ldots$ & 1998 Nov 19 & 51,136 & $82 \mathrm{~S}$ & 286.54 & 0.01 & 11 & 534 & 99.6 & 206.1 & -11.1 & 1.41 & $\mathrm{~N}$ \\
\hline $297 \ldots \ldots \ldots \ldots \ldots$ & 1998 Nov 22 & 51,139 & $82 \mathrm{O}$ & 92.04 & 0.04 & 11 & 203 & 36.7 & 206.0 & -11.0 & 1.47 & $\mathrm{Y}$ \\
\hline 307................... & 1998 Nov 23 & 51,140 & $82 \mathrm{~N}$ & 318.93 & 0.01 & 11 & 186 & 33.4 & 212.4 & 0.0 & 1.21 & $\mathrm{~N}$ \\
\hline 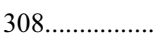 & 1998 Nov 23 & 51,140 & $10 \mathrm{~N}$ & 271.30 & 0.01 & 11 & 216 & 39.1 & 216.0 & 4.2 & 1.24 & $\mathrm{Y}$ \\
\hline $994 \ldots \ldots \ldots \ldots . . . .$. & 1999 Oct 6 & 51,457 & $76 \mathrm{~S}$ & 275.04 & 15.00 & 11 & 133 & 23.4 & 209.9 & -5.0 & 1.84 & $\mathrm{~N}$ \\
\hline $1923 \ldots \ldots \ldots \ldots$ & 2000 Dec 8 & 51,886 & $0 \mathrm{O}$ & 215.00 & 44.99 & 29 & 71 & 8.2 & 86.4 & 0.0 & 1.75 & $\mathrm{U}$ \\
\hline $1924 \ldots \ldots \ldots \ldots . .$. & 2000 Dec 8 & 51,886 & $0 \mathrm{O}$ & 354.00 & 45.99 & 34 & 78 & 8.6 & 168.6 & 11.9 & 1.88 & $\mathrm{U}$ \\
\hline $1925 \ldots \ldots \ldots \ldots$ & 2000 Dec 8 & 51,886 & $0 \mathrm{O}$ & 349.98 & 45.78 & 17 & 48 & 6.1 & 165.3 & 6.6 & 1.60 & $\mathrm{U}$ \\
\hline $2955 \ldots \ldots \ldots \ldots . . .$. & 2002 Feb 7 & 52,312 & $82 \mathrm{~N}$ & 9.82 & 0.00 & 21 & 166 & 27.7 & 209.3 & -4.5 & 2.07 & $\mathrm{Y}$ \\
\hline $2960 \ldots \ldots \ldots \ldots . . . .$. & 2002 Feb 8 & 52,313 & $82 \mathrm{~S}$ & 25.79 & 0.01 & 11 & 127 & 22.2 & 208.0 & -7.4 & 1.16 & $\mathrm{Y}$ \\
\hline $2968 \ldots \ldots \ldots \ldots . . .$. & 2002 Feb 9 & 52,314 & $82 \mathrm{~N}$ & 90.64 & 0.00 & 19 & 94 & 14.4 & 207.8 & -7.3 & 1.68 & $\mathrm{Y}$ \\
\hline $3511 \ldots \ldots \ldots \ldots . . .$. & 2002 Dec 6 & 52,614 & $0 \mathrm{O}$ & 65.19 & 90.14 & 14 & 89 & 14.4 & 163.5 & -8.2 & 1.17 & $\mathrm{Y}$ \\
\hline $3512 \ldots \ldots \ldots \ldots$ & 2002 Dec 6 & 52,614 & $0 \mathrm{O}$ & 335.41 & 25.32 & 11 & 90 & 15.2 & 177.3 & -9.3 & 1.22 & $\mathrm{Y}$ \\
\hline $3557 \ldots \ldots \ldots \ldots . . .$. & 2002 Dec 31 & 52,639 & $0 \mathrm{O}$ & 65.50 & 89.96 & 11 & 87 & 14.6 & 165.6 & -10.5 & 1.26 & $\mathrm{Y}$ \\
\hline $3559 \ldots \ldots \ldots \ldots$ & 2002 Dec 31 & 52,639 & $0 \mathrm{O}$ & 335.41 & 25.31 & 11 & 86 & 14.4 & 176.4 & -9.9 & 1.20 & $\mathrm{Y}$ \\
\hline 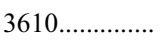 & 2003 Jan 27 & 52,666 & $61 \mathrm{~N}$ & 274.99 & 52.50 & 17 & 148 & 25.1 & 139.0 & -10.8 & 1.63 & $\mathrm{Y}$ \\
\hline $3628 \ldots \ldots \ldots \ldots . .$. & 2003 Jan 28 & 52,667 & $61 \mathrm{~S}$ & 275.01 & 52.52 & 17 & 165 & 28.3 & 137.3 & -10.9 & 0.97 & $\mathrm{Y}$ \\
\hline $3629 \ldots \ldots \ldots \ldots$ & 2003 Jan 28 & 52,667 & $61 \mathrm{~N}$ & 275.00 & 52.50 & 11 & 129 & 22.6 & 142.0 & -10.9 & 0.97 & $\mathrm{Y}$ \\
\hline $3634 \ldots \ldots \ldots \ldots$ & 2003 Jan 29 & 52,668 & $62 \mathrm{~N}$ & 275.00 & 50.01 & 18 & 162 & 27.5 & 159.1 & -13.0 & 1.04 & $\mathrm{Y}$ \\
\hline $3642 \ldots \ldots \ldots \ldots . .$. & 2003 Feb 1 & 52,671 & $62 \mathrm{~S}$ & 274.99 & 49.99 & 11 & 97 & 16.5 & 135.6 & -13.2 & 1.73 & $\mathrm{Y}$ \\
\hline $3643 \ldots \ldots \ldots \ldots$ & 2003 Feb 1 & 52,671 & $62 \mathrm{~S}$ & 274.99 & 50.00 & 11 & 119 & 20.7 & 159.1 & -13.2 & 1.68 & $\mathrm{Y}$ \\
\hline $4114 \ldots \ldots \ldots \ldots$ & 2003 Sep 20 & 52,902 & $0 \mathrm{~S}$ & 309.49 & 90.02 & 41 & 127 & 16.5 & 80.1 & 0.0 & 1.59 & $\mathrm{Y}$ \\
\hline $4115 \ldots \ldots \ldots \ldots$ & 2003 Sep 20 & 52,902 & OS & 309.34 & 90.15 & 11 & 69 & 11.2 & 78.7 & 0.0 & 1.36 & $\mathrm{Y}$ \\
\hline 4116.................... & 2003 Sep 20 & 52,902 & $0 \mathrm{O}$ & 219.49 & 41.40 & 11 & 77 & 12.7 & 82.1 & 0.0 & 1.40 & $\mathrm{Y}$ \\
\hline $4119 \ldots \ldots \ldots \ldots$ & 2003 Sep 20 & 52,902 & $0 \mathrm{O}$ & 309.40 & 90.08 & 11 & 70 & 11.4 & 79.8 & 0.0 & 1.71 & $\mathrm{Y}$ \\
\hline
\end{tabular}

Notes.-Col. (1): SDSS run number. Col. (2): UT Date. Col. (3): UT MJD. Col. (4): Survey strip number; 82 and 10 are on the celestial equator. N = north, $\mathrm{S}=$ south, $\mathrm{O}=$ other (non-survey strips). Cols. $(5,6)$ : J2000 node and inclination of stripe great circle. Col. (7): Start field for run; in some cases ramp-up takes more than 11 fields. Col. (8): End field. Col. (9): Gross area in square degrees. Cols. $(10,11)$ : Galactic coordinates of closest approach to the Galactic plane of any field center. Col. (12): Median PSF FWHM ( $r$-band, camera column 3). Col. (13): Photometric? ( $Y=$ yes, $N=$ no, $U=$ unkown). Run 273 has an aperture obstruction early in the run, and run 307 is slightly cloudy near the end. Data in the 1998-1999 runs are now public.

for proper scientific use of the data. A complete description of the data outputs can be obtained at the Web site mentioned above, at the SDSS DR2 Web site, ${ }^{44}$ and in papers by Stoughton et al. (2002) and Abazajian et al. $(2003,2004)$.

${ }^{44}$ See http://www.sdss.org.
The frames pipeline produces a catalog of objects (fpObjc files) described by instrumental quantities: $(x, y)$-positions on a frame, CCD counts, and radii. Astrometric calibrations are applied by the astrometric pipeline (astrom) and are accurate to better than $0^{\prime \prime} 1 \mathrm{rms}$ in each coordinate (Pier et al. 2003). Because early commissioning data sometimes had poor telescope

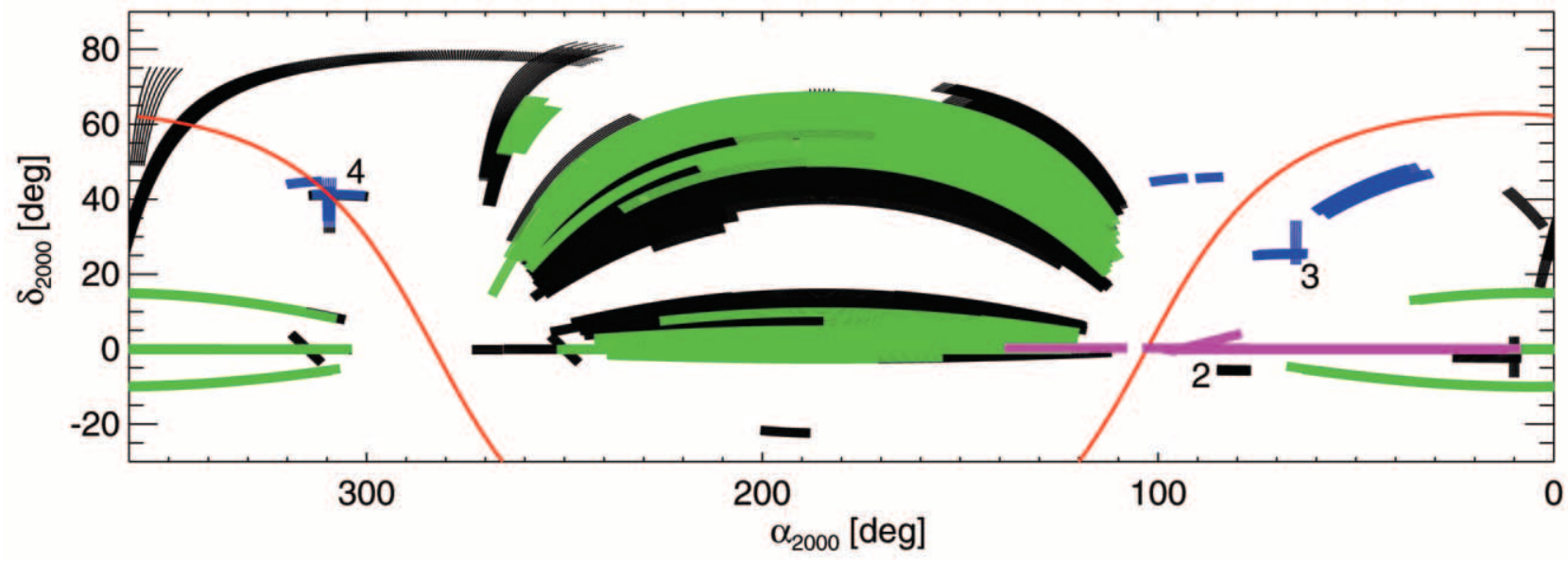

FIG. 1.-The SDSS footprint in equatorial coordinates, consisting of the (currently released) Orion data (magenta), other Galactic plane runs (blue), SDSS DR2 (green), and other SDSS data taken through 2003 December (black). Numbers mark the regions shown in Figs. 2-4. 

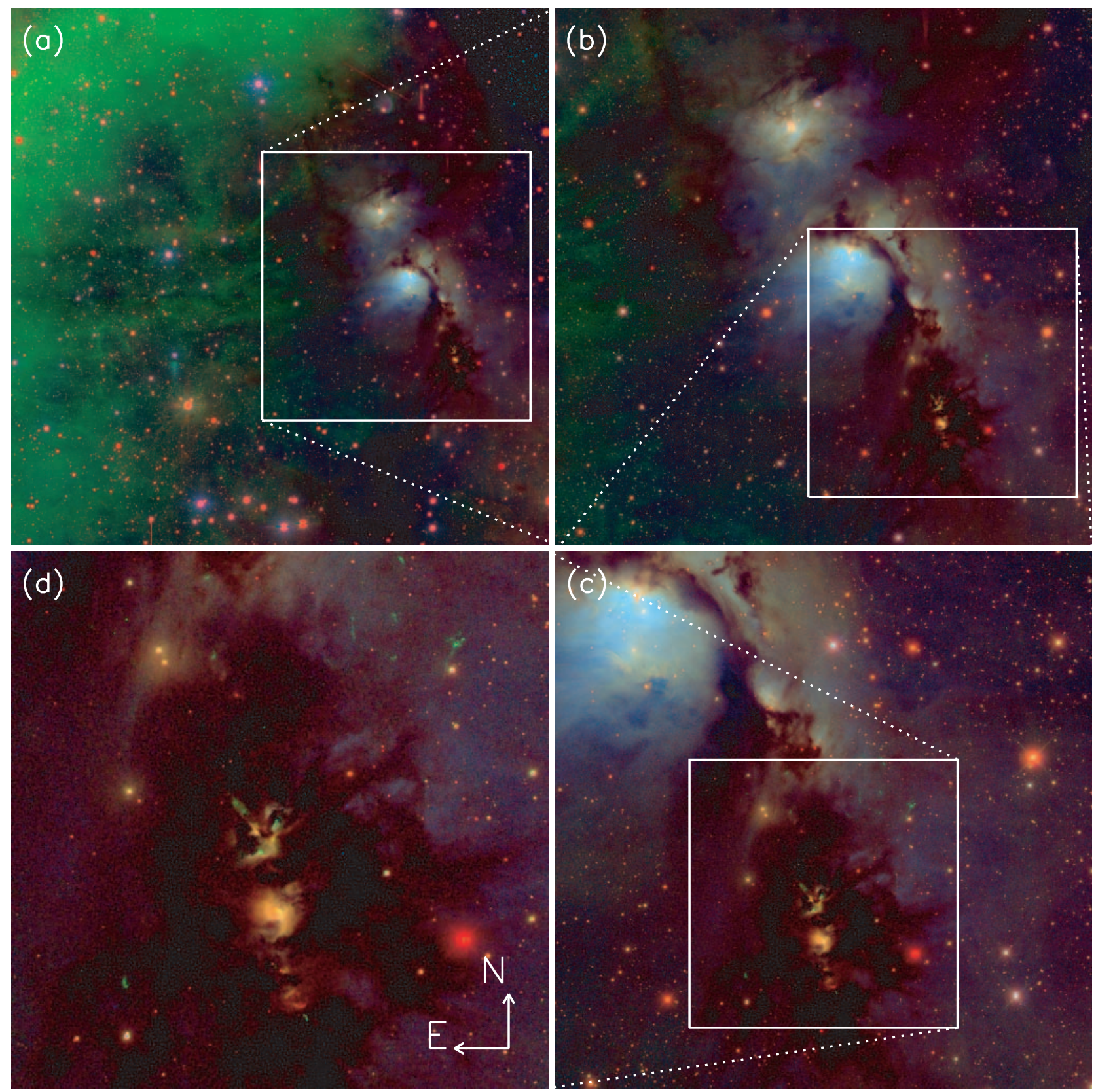

FIG. 2.-Orion: A section of runs 259 and 273 centered at $\mathrm{J} 2000(\alpha, \delta)=\left(05^{\mathrm{h}} 48^{\mathrm{m}} 28^{\mathrm{s}},+00^{\circ} 04^{\prime} 48^{\prime \prime}\right)$. The red, green, and blue color planes represent the SDSS $i, r$, and $g$ filters, respectively, so that $\mathrm{H} \alpha$ emission appears green. Celestial north is indicated in $(d)$. The widths of $(a-d)$ are $108^{\prime}, 54^{\prime}, 27^{\prime}$, and 13.5 , respectively. The nebulae NGC 2071 (upper) and NGC 2068 are visible in (b), and (d) zooms in on the object HH 24 (upper middle).

pointing information (pointing errors of up to 0.5 ), astrometric preprocessing using the discrete cross-correlation method described by Hogg et al. (2001) has been performed to allow such data to be processed in an automated fashion. The flat-fielding and photometric calibration were derived from a global recalibration using all the available repeat imaging (Schlegel et al. 2004). The zero points of the photometric solutions are forced to agree on average with the calibrations derived from the Photometric Telescope (Fukugita et al. 1996; York et al. 2000; Smith et al. 2002) used in SDSS DR2.

\subsubsection{Automation with photoop}

The importance of a completely automated data processing pipeline cannot be overemphasized; it ensures that all the reductions are carried out in a homogeneous fashion and protects against human biases. Furthermore, it ensures that all reductions are perfectly reproducible, allowing comparisons between different reduction attempts. This is a necessary prerequisite for a survey such as SDSS that targets subsamples of galaxies for follow-up observations, because it enables one to measure the completeness of these subsamples even as the underlying software evolves.

While the standard SDSS pipelines perform well within the standard survey region, the radically different characteristics of the data near the Galactic plane require adjustments of various pipeline parameters to process these data. In order to implement these in an automated manner, we have developed a meta-pipeline, phot oop, to manage all aspects of processing. In production mode, the only human intervention required is to specify the runs to be processed; after that, photoop sets up 

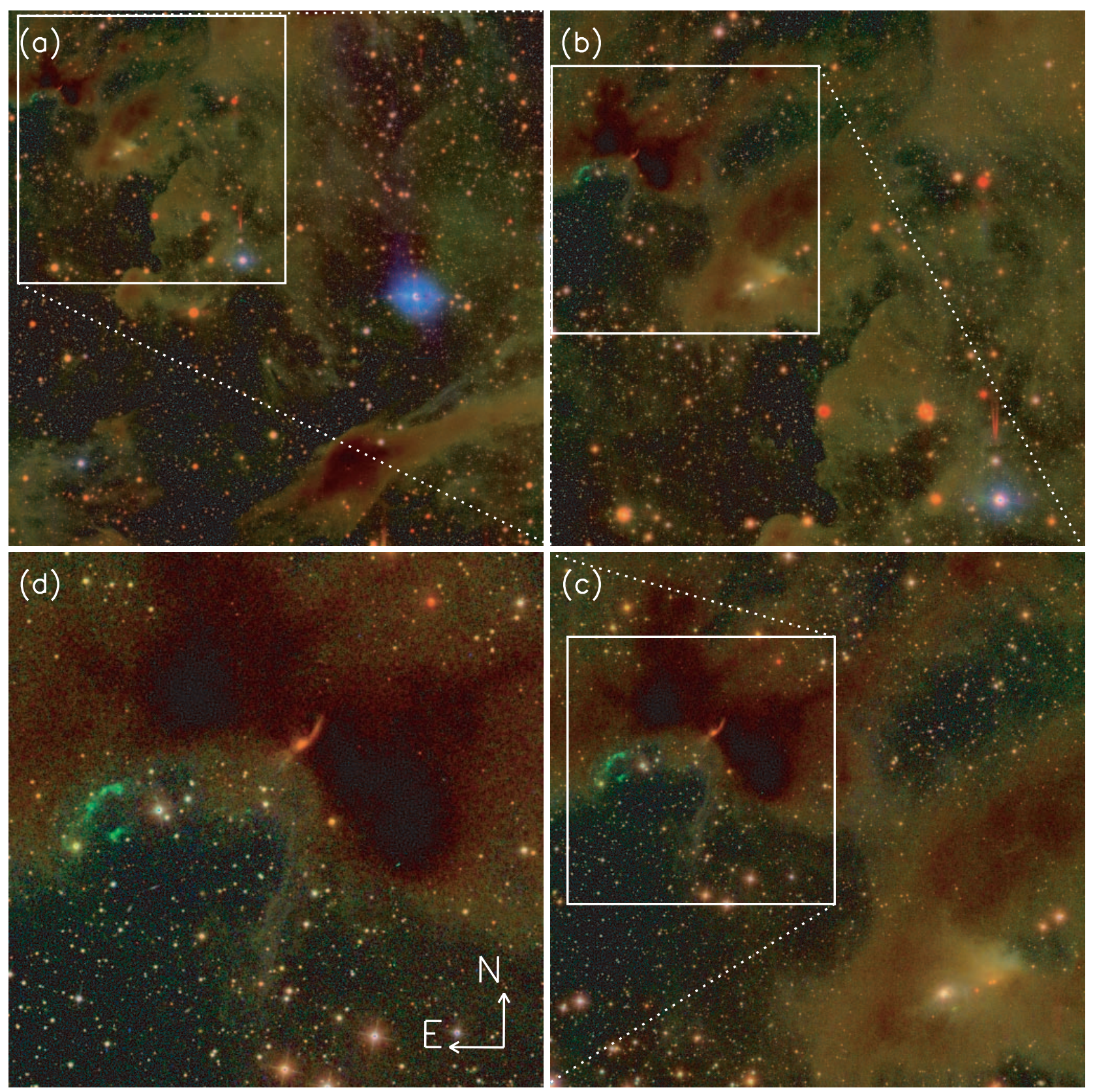

FIG. 3.-Taurus: A section of runs 3512 and 3559 centered at $(\alpha, \delta)=\left(04^{\mathrm{h}} 24^{\mathrm{m}} 40^{\mathrm{s}},+25^{\circ} 41^{\prime} 24^{\prime \prime}\right)$. The color scheme and scale are identical to Fig. 2. The large dark cloud in the lower right of $(a)$ is Barnard 215, which joins with Lynds 1506 further to the right; $(d)$ zooms in on HH 31A-31D and associated outflows (middle left).

the photometric pipeline and generates the required input data for each run, including flat fields, astrometric catalogs, and configuration files. Perl scripts then manage the actual processing across several Unix servers with a shared network file system. Other photoop scripts automate daily maintenance tasks: monitoring the processing status of each run, posting results to an internal Web page, and testing data integrity. Notices of pipeline failures and data-integrity warnings are automatically sent by E-mail to the responsible parties. Furthermore, photoop has been designed to be portable so that anyone with sufficient computing power and access to the raw data can replicate the entire processing system.

\subsubsection{Processing Software Versions and Reruns}

As the SDSS software pipelines have evolved over time, it has been necessary to keep track of the various processing attempts with rerun numbers. Data processing done by means of photoop is strictly versioned, with rerun numbers above 100 to avoid confusion with the main survey rerun numbers (0-99). There is a one-to-one correspondence between rerun number and software versions. The current rerun as of this writing is 137 , corresponding to ssc v5_3_4, astrom v3_7, photo v5_4_25, and v4 flat fields and photoop v1_0. For historical reasons, the psp and frames steps of the pipeline are both part of the photo software product. The instructions below for accessing and using the data refer to rerun 137 . Future refinements of the software will have higher rerun numbers and will be announced on the Web site.

\subsubsection{Calibration}

Survey data releases are indirectly calibrated to a set of primary photometric standard stars measured with the US Naval 

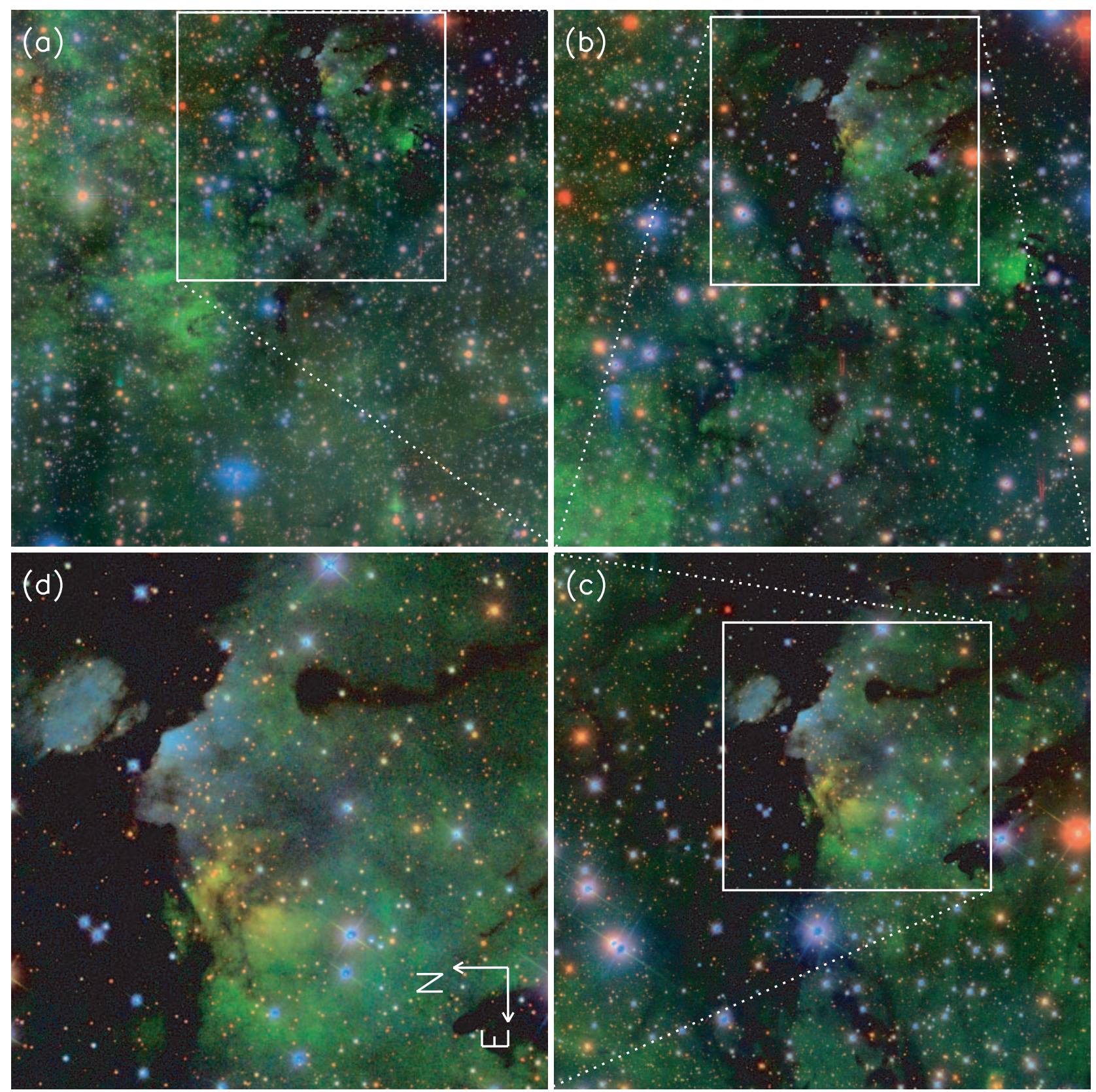

FIg. 4.-Cygnus: A section of runs 4115 and 4119 centered at $(\alpha, \delta)=\left(20^{\mathrm{h}} 36^{\mathrm{m}} 04^{\mathrm{s}},+40^{\circ} 26^{\prime} 24^{\prime \prime}\right)$. The color scheme and scale are identical to Fig. 2 . The $\mathrm{H}$ II region LBN 271 is visible in (a) (middle left), and (d) zooms in on a beautiful complex of dark clouds in front of LBN 258.

Observatory (USNO) $1 \mathrm{~m}$ telescope in the SDSS filters. This photometric system is denoted by $u^{\prime} g^{\prime} r^{\prime} i^{\prime} z^{\prime}$ and is defined by Smith et al. (2002). These primary standards are bright enough to saturate the $2.5 \mathrm{~m}$ survey telescope, so the photometric system is transferred to a set of secondary standard star "patches" observed by the $0.5 \mathrm{~m}$ "Photometric Telescope" (PT) situated adjacent to the survey telescope. These secondary standards are used to calibrate the $2.5 \mathrm{~m}$ native filter system (denoted ugriz) to the USNO system for typical star colors. Note that the $u^{\prime} g^{\prime} r^{\prime} i^{\prime} z^{\prime}$ and ugriz systems are significantly different (see, e.g., Stoughton et al. 2002). PT patches are sparse or nonexistent for much of the Orion region, so we have instead used the übercalibration algorithm (Schlegel et al. 2004) to tie the eight Orion runs together photometrically. This algorithm lets the calibration zero points in each CCD ( $a$-terms) and atmospheric extinction ( $k$-terms) float night by night, constrained by multiple observations of stars in run overlaps. Run 308 does not overlap any of the other Orion runs and is therefore calibrated using the same $a$ - and $k$-terms as run 307 from the same night. Übercalibration minimizes the rms magnitude residuals in repeat observations of $\sim 10^{6}$ stars by adjusting these $a$ - and $k$-terms. Because the north and south strips of the equatorial stripe overlap only at the camera column edges, and the flat fields are less certain there, it is necessary to use a perpendicular scan (run 2766) to tie the 12 independent camera columns together. This run is connected to the Orion runs by means of four other equatorial runs $(94,125,1755,2677)$. For equatorial drift scans the air mass is constant, making the $a$ - and $k$-terms degenerate, so $k$-terms are fixed to canonical values.

Most stars are observed multiple times by this set of runs, and for each star the residual between each run and the mean of all observations of that star is shown in Figure 5. Only stars 

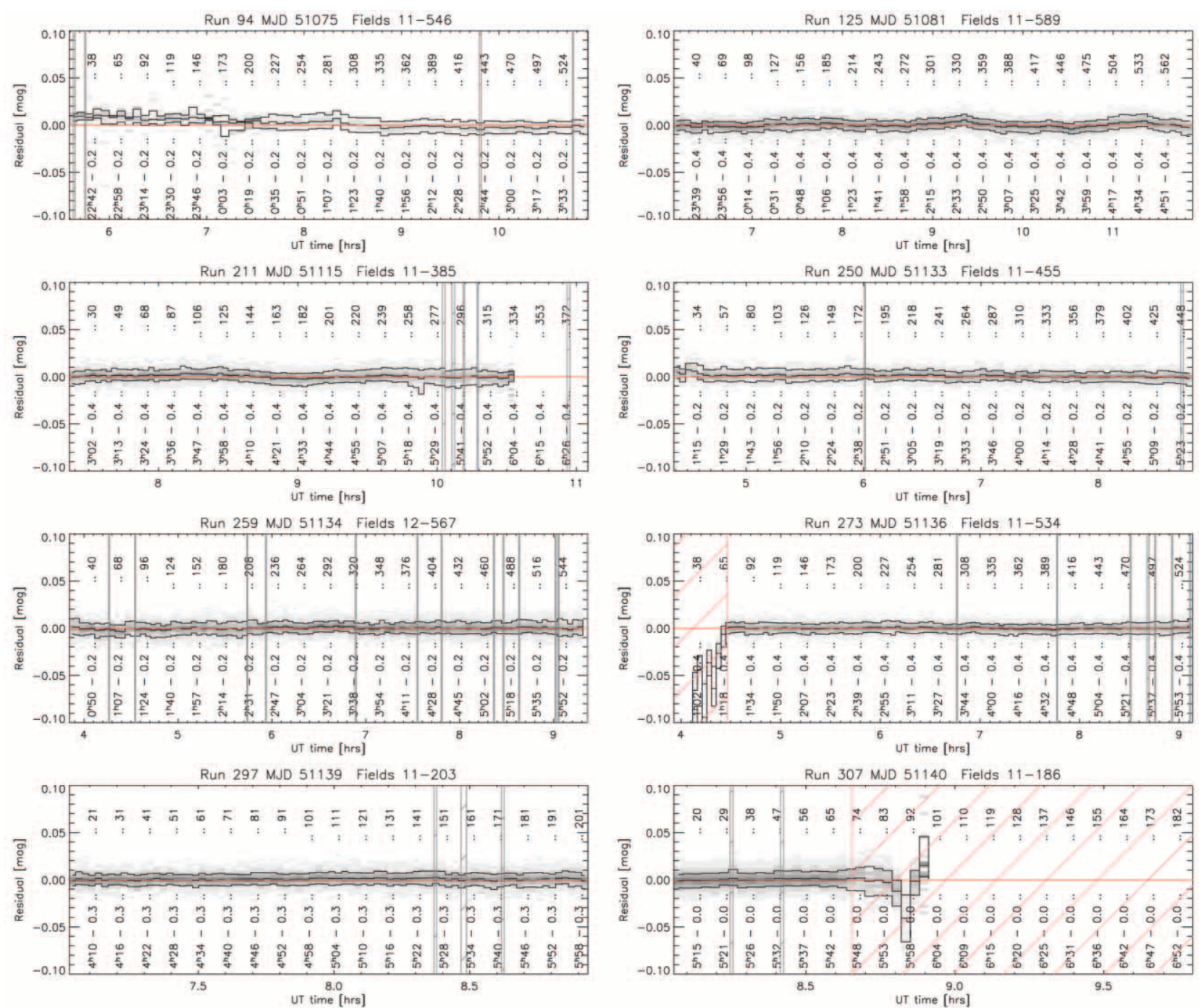

Run 994 MJD 51457 Fields 11-133
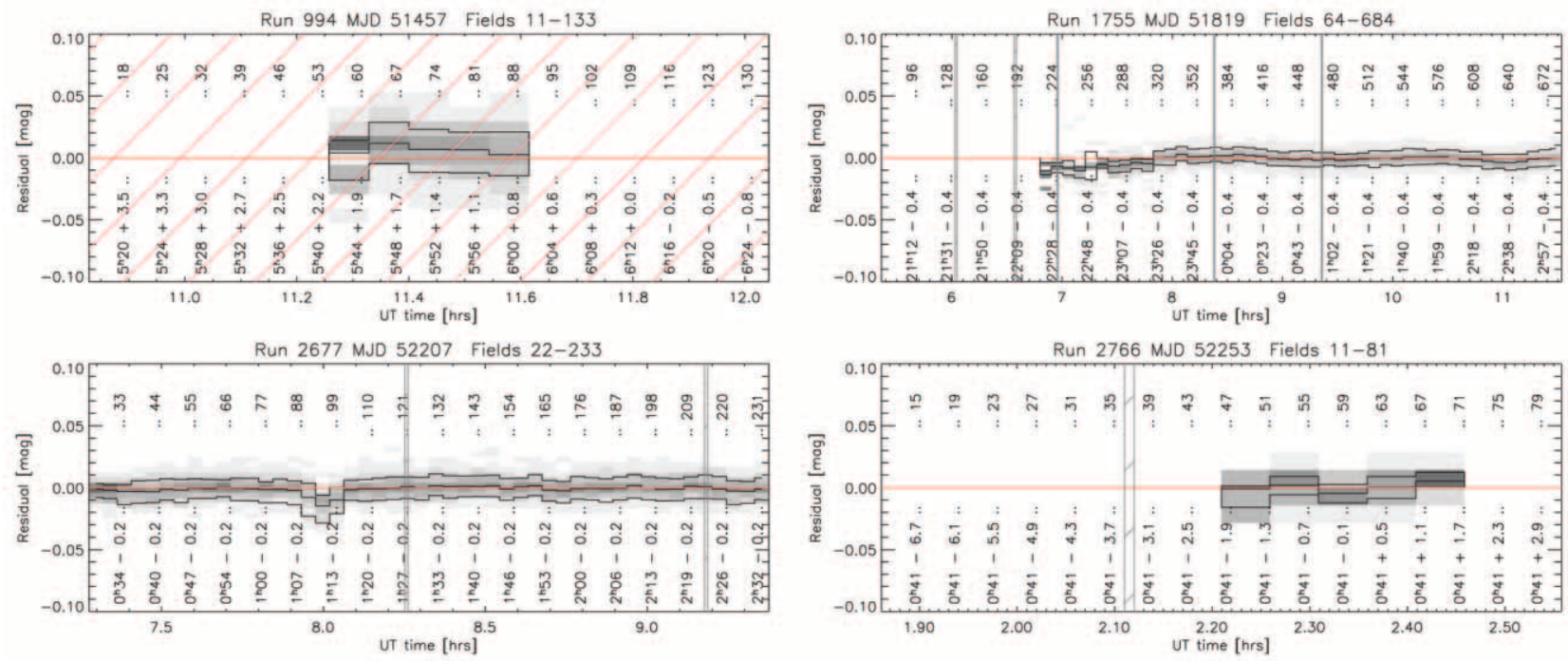

FIG. 5.-Measured magnitudes minus means for each star of each run used in the Orion übercalibration (see $\S 2.2 .3$ ). Stars brighter than 19.0, 19.0, 19.0, 18.5, and $17.0 \mathrm{mag}$ in $u, g, r, i$, and $z$ respectively are used in the calibration; results are shown for the $r$ band only. Differences are represented as gray scales with 25 th, 50th, and 75th percentile lines. Results are similar for $g$ and $i$ bands, with more photometric scatter in $u$ and $z$ (see Fig. 6). Cloudy or otherwise unphotometric data are indicated by red hatches, and gray hatches indicate fields where the pipeline was unable to determine a good point-spread function; these data are not used in fits. Run 308 does not appear here, because it has no overlap with the others, but it is calibrated by fixing its parameters to run 307 . 

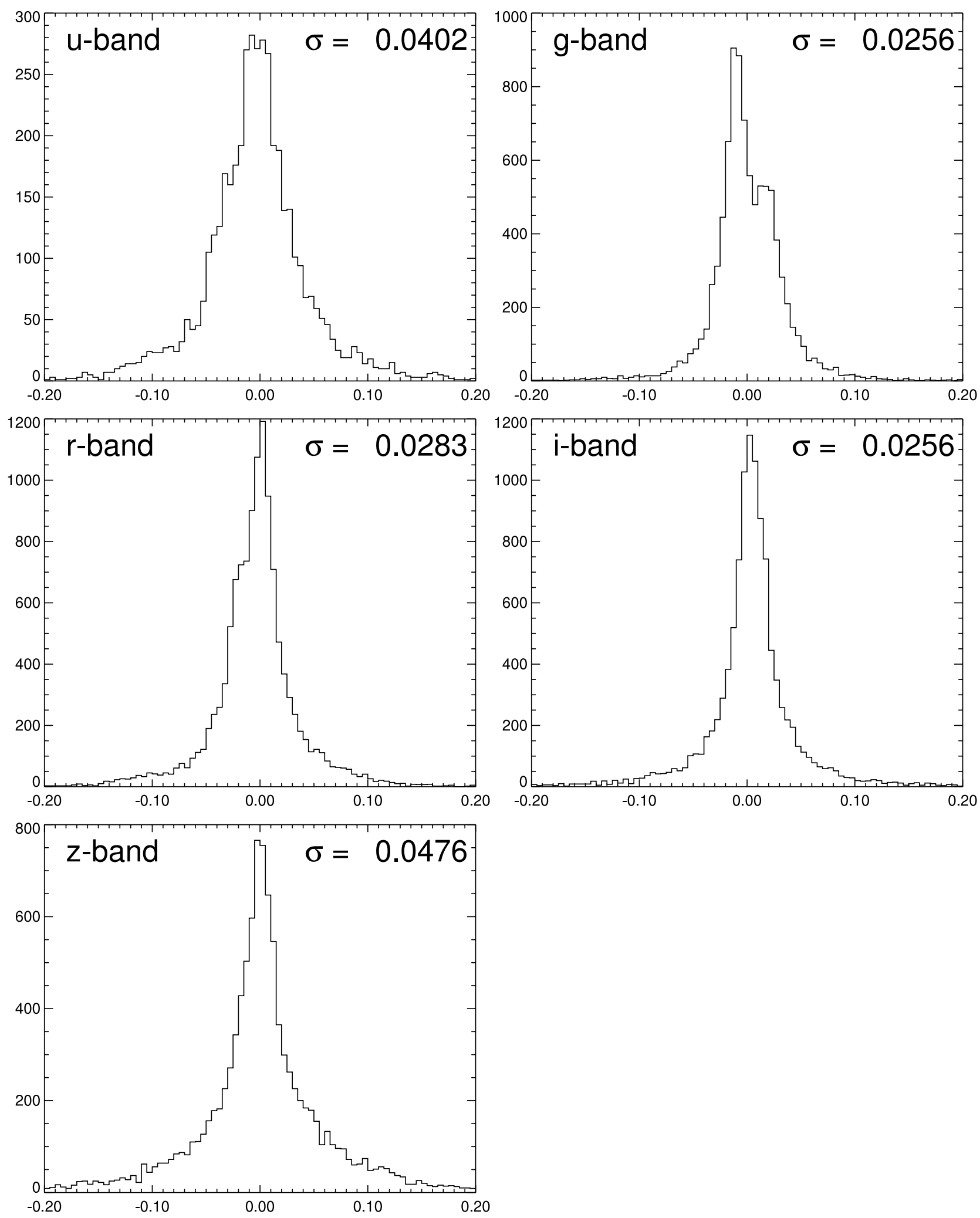

Fig. 6. - Histograms of aperture magnitude differences between runs 259 and 297 for the five SDSS bands. Stars with 7 ".43 radius aperture magnitudes brighter than 19.0, 19.0, 19.0, 18.5, and 17.0 in $u, g, r, i$, and $z$ are shown. Note that the measurement uncertainty in each run is a factor of $\sqrt{2}$ smaller than the histogram width. The bimodality in the $g$ band is likely caused by $1 \%-2 \%$ variation in the flat fields with time. 
TABLE 2

CVS PRoducts

\begin{tabular}{|c|c|c|c|c|}
\hline Product & CVS Version & Language & Analysis? & Description \\
\hline dust .................. & v0_0 & IDL/Fortran/C & Opt. & The Schlegel et al. (1998) $E(B-V)$ extinction maps \\
\hline eups ....... & v0_4 & Perl & Opt. & Product management software \\
\hline 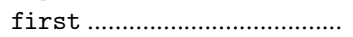 & v03Apr11 & $\ldots$ & Opt. & FIRST (Becker et al. 1995) radio catalog \\
\hline idlutils................................. & v5_0_0 & IDL/Fortran/C & Yes & General IDL tools; required for all IDL tasks \\
\hline 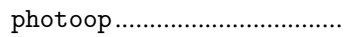 & v1_1 & Perl/IDL & Yes & SDSS Perl/IDL tools \\
\hline 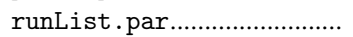 & $\ldots$ & $\ldots$ & Yes & The list of runs processed, and their locations \\
\hline 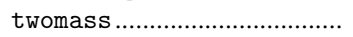 & allsky & $\ldots$ & Opt. & 2MASS (Cutri et al. 2003) Point Source Catalog \\
\hline 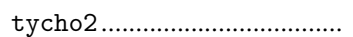 & v0_0 & $\ldots$ & Opt. & Tycho (Høg et al. 2000) astrometric catalog \\
\hline (n....................... & v2_final & $\ldots$ & Opt. & UCAC (Zacharias et al. 2000) astrometric catalog \\
\hline usno .......... & vB & $\ldots$ & Opt. & USNO-B (Monet et al. 2003) astrometric catalog \\
\hline
\end{tabular}

NoтE.- The list of all products used for either processing, analyzing, or accessing the data. We limit ourselves to those products unique to this reduction; the reader is referred to Stoughton et al. (2002) for the official survey pipeline code. The column "Analysis" marks products either necessary ("Yes") or optional ("Opt.") for data analysis.

with 7".43 radius aperture magnitudes brighter than 19.0, 19.0, 19.0, 18.5, and 17.0 in $u, g, r, i$, and $z$ respectively were used for the calibration, and the resulting difference histograms have a $5 \sigma$ clipped rms of 40, 26, 28, 26, and 48 mmag (Fig. 6). This calibration method will be described in detail elsewhere (Schlegel et al. 2004), and astrophysical tests of its accuracy will be provided in Finkbeiner et al. (2004).

\subsection{Public Data Access}

Table 2 lists all software and ancillary data products (in addition to the standard survey software described by Stoughton et al. 2002) that are used in the data processing, access, and analysis steps. These products are organized with the Concurrent Versions System (CVS). ${ }^{45}$ IDL $^{46}$ routines for displaying and calibrating images, extracting photometric parameters for sources, and matching sources to other public catalogs are included. Recipes using some of the most powerful routines are given in examples below. We refer the reader to the Web site for details on downloading and installing these packages; here we limit ourselves to brief descriptions of the data products that make up this data release.

This data release contains three data sets: calibrated images for every field, object catalogs for every field, and trimmed catalogs of "stars" (all point sources, including quasars) and "galaxies" (extended sources) for every run. The images have had cosmic rays removed, had CCD defects corrected, and been flat-fielded and photometrically calibrated. In addition, these images have accurate astrometry stored in a FITS-compliant header and therefore do not depend on any auxiliary information. The object catalogs (calibObj) include all data fields in the uncalibrated $\mathrm{fpObjc}$ catalogs produced by the SDSS frames pipeline, as well as calibrated quantities. Object fluxes are calibrated, and the CCD positions of objects are translated to equatorial coordinates using the best-fit astrometric solution from the SDSS astrom pipeline. An overview of the differences between these calibObj catalogs and the standard SDSS tsObj files (available at the Data Archive Server) ${ }^{47}$ is given in $\S 3$, and the calibObj format is described in detail in Appendix A.

The calibObj catalogs for the eight public runs require 50 gigabytes. For users who prefer data in a more compact format, we also provide trimmed stellar and galaxy catalogs

\footnotetext{
${ }^{45}$ See http://www.cvshome.org.

${ }_{46} \mathrm{See}$ http://www.rsinc.com.

47 See http://das.sdss.org/DR2/data/imaging/; see also Stoughton et al. (2002) for more details.
}

for each camera column in each of the eight runs, which total 1.5 Gbyte for the stars and 2.5 Gbyte for the galaxies. The trimmed catalogs contain all stars with any of $u, g, r, i$, and $z$ brighter than $22.5,22.5,22.5,22.0$, and $21.5 \mathrm{mag}$, respectively, and galaxies brighter than 21.0,22.0,22.0, 20.5, and $20.1 \mathrm{mag}$. These cuts were made after applying the Schlegel et al. (1998, hereafter SFD98) extinction correction for the purpose of object selection - the extinction correction is not applied to the flux values in the catalogs. These trimmed star and galaxy catalogs are a strict subset of the quantities described in Appendix A and contain no additional information. The list of fields contained is given on the Web site.

Instructions for downloading any of these data are at the Web site. We advise users to organize downloaded data using the directory structure described there, as this ensures compatibility with our released software.

\subsection{Release Schedule}

Runs 211 through 994, comprising 470 square degrees, are now available to the public at the Web site. Further data will be released on a schedule that approximately parallels that of the SDSS data releases. We plan to release runs through 4119 at the time of SDSS Data Release 4, expected in 2005. Note that these data, unlike the SDSS data releases, contain no spectra.

\section{DATA FORMATS}

The Galactic plane data processing and data formats differ somewhat from those of SDSS DR2, partly from changes necessary to process these data, and partly to rationalize some naming conventions. The catalogs produced by the current data processing are in calibObj files, whose format we now describe.

1. All data fields output by the frames step of the photometric pipeline in $\mathrm{fpObjc}$ files are retained, and not overwritten as they are in $t s 0 b j$ files.

2. Object fluxes are given in linear units, instead of the asinh magnitudes (or "luptitudes"; Lupton et al. 1999) used in the other data releases. This facilitates, for example, co-adding of repeat imaging at the catalog level.

3. Calibrated quantities are presented in nanomaggies (abbreviated nMgy, a flux density), where $1 \mathrm{Mgy}$ is the $\mathrm{AB}$ flux density of a zeroth-magnitude flat-spectrum object (Oke \& Gunn 1983). An AB magnitude of 22.5 corresponds to $1 \mathrm{nMgy}$ in any filter. The approximate conversion to physical units is 


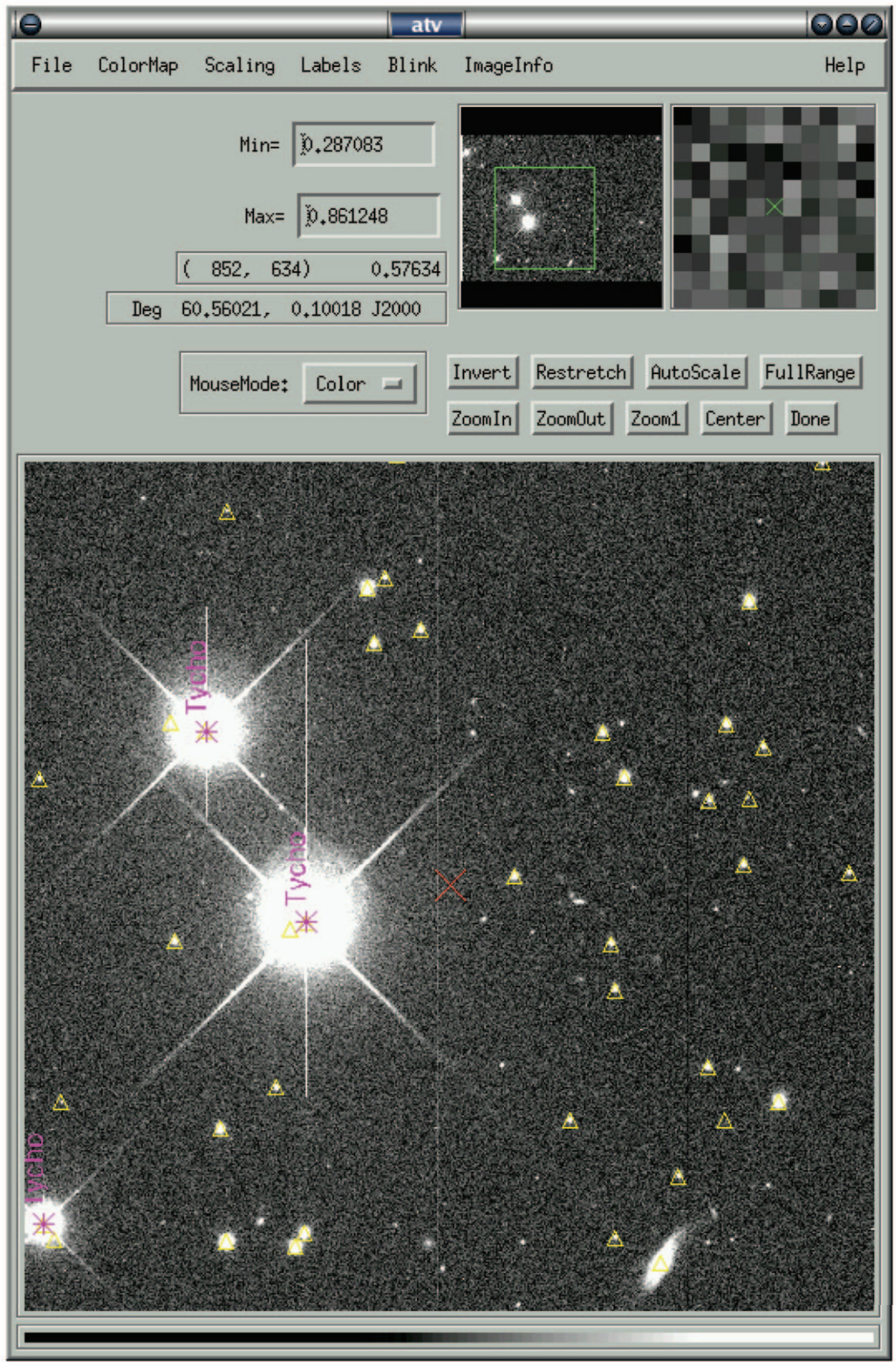

FIG. 7.- The display tool atvsdss. Here it finds a raw SDSS image at the given coordinates, flat-fields and calibrates on the fly, matches and overplots Tycho stars (magenta) and 2MASS stars (yellow triangles), and displays a red cross at the requested coordinates. See $\S 4.1$ for more details. 
$1 \mathrm{Mgy}=3631 \mathrm{Jy}$, where $1 \mathrm{Jy}=10^{-26} \mathrm{~W} \mathrm{~m}^{-2} \mathrm{~Hz}^{-1}=10^{-23} \mathrm{ergs}$ $\mathrm{s}^{-1} \mathrm{~cm}^{-2} \mathrm{~Hz}^{-1}$. This conversion is only accurate to about $5 \%$ (Oke \& Gunn 1983), although relative zero-point offsets among the SDSS filters are determined to $1 \%-2 \%$ (Abazajian et al. 2004). Note that the conversion of a measured calibrated magnitude of a source in a broadband filter system to a physical flux density depends on the spectral shape of the source, so precise conversion factors do not exist.

4. Names are rationalized. In $\mathrm{fpObjc} \mathrm{files,} \mathrm{for} \mathrm{historical} \mathrm{rea-}$ sons, model counts are known as COUNTS_MODEL and Petrosian counts as PETROCOUNTS. In the standard survey tsObj files these fields are overwritten with calibrated magnitudes, causing confusion. In the calibObj data structure, these uncalibrated data fields appear unchanged, with the corresponding calibrated quantities appended as MODELFLUX and PETROFLUX.

5. Flux uncertainties are nearly Gaussian in the low signal-tonoise limit (unlike errors in magnitudes). We express these errors as an inverse variance ("ivar"). This is convenient for inversevariance weighting when combining quantities and handles zero signal-to-noise ratio (ivar $=0$ ) gracefully, as demonstrated in Appendix B. Names are, for example, PETROFLUX_IVAR.

6. Extinction in magnitudes (from SFD98) is given in the five SDSS bands and is called EXTINCTION, not REDDENING as in the tsObj files. These values are calculated assuming the standard $R_{V}=3.1$ reddening law (Cardelli et al. 1989), which may be inappropriate for some low-latitude regions.

7. Model profile angles such as PHI_ISO_DEG, PHI_DEV_ DEG, and PHI_EXP_DEG are expressed as angles in degrees east of north.

8. PSF_FWHM (arcseconds) is given for every object in each band. Because of the telescope optics and seeing, the PSF varies across a frame. The PSF is modeled by a set of eigenfunctions measured by bright stars in the frame, and the interpolated PSF calculated at the position of every object detected by photo. Note that this same information is available through the adaptive moments of the reconstructed PSF.

9. Wherever possible, objects are matched to the FIRST radio catalog (Becker et al. 1995), the 2MASS Point Source Catalog (Cutri et al. 2003), and the USNO-B astrometric survey (Monet et al. 2003).

\section{SCIENCE}

The science enabled by the wide-area imaging reported here generally lies in the areas of Galactic structure, star formation, and interstellar matter. Several examples are noted here; for the simplest of these, we also provide fragments of IDL code that illustrate the functionality of our publicly available software (Table 2).

\subsection{Displaying an Image}

Using the photoop tools, one can calibrate the raw image data (idR files) at read time and eliminate the need to store a second, calibrated copy of the image data (Fig. 7). Following is a sequence of IDL commands to find (run, camcol, field) for a position on the sky, read in a calibrated image with astrometric information, match objects in the field to the 2MASS catalog, and display the image with matches overplotted in the IDL display tool atv (Barth 2001).

; To see which runs cover $R A=60.56$, dec $=0.1$ IDL $>$ imlist=sdss_findimage $(60.56,0.1$, rerun=137, \$ /print)

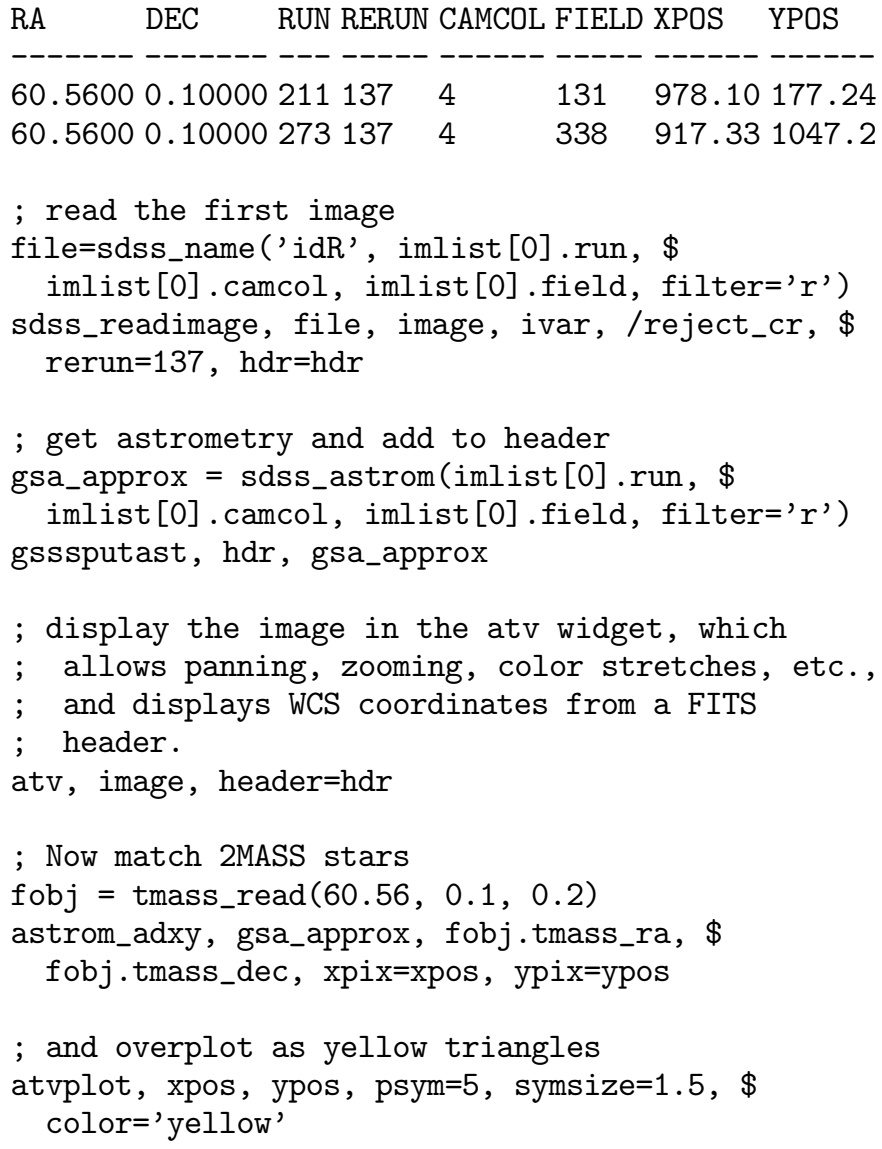

The above commands demonstrate the power of some of the tools available. The atv wrapper atvsdss does all of the above with the simple command

atvsdss, ra $=60.56$, dec=0.1, /catalog, rerun=137

where the rerun number is required for use of the survey astrom astrometric solutions, and / catalog matches and overplots Tycho stars (Fig. 7, magenta), 2MASS stars (yellow triangles), and a cross at the requested coordinates (red).

\subsection{The Stellar g-r-i Color Diagram}

The following is a simple example that plots the stellar locus in the $g-r-i$ color plane and provides an introduction to using the catalog data. We start by reading in all the objects from a segment of a run and then select only the stars with reliable photometry using the object flags. We then demonstrate the conversion of calibrated fluxes into magnitudes and use these to construct a magnitude-limited sample of stars. Finally, we compute and plot the $g-r$ and $r-i$ colors of these stars. The output of this code is shown in Figure 8.

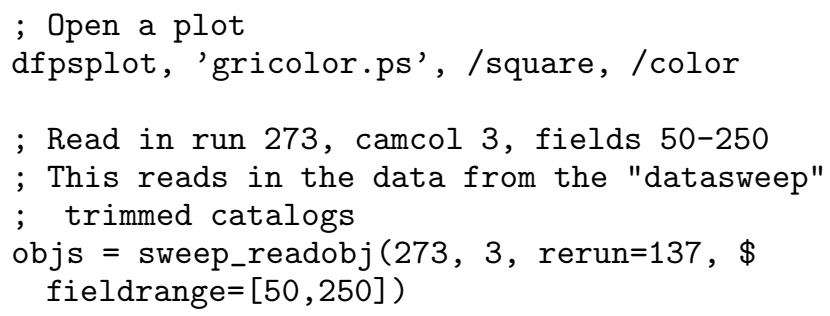




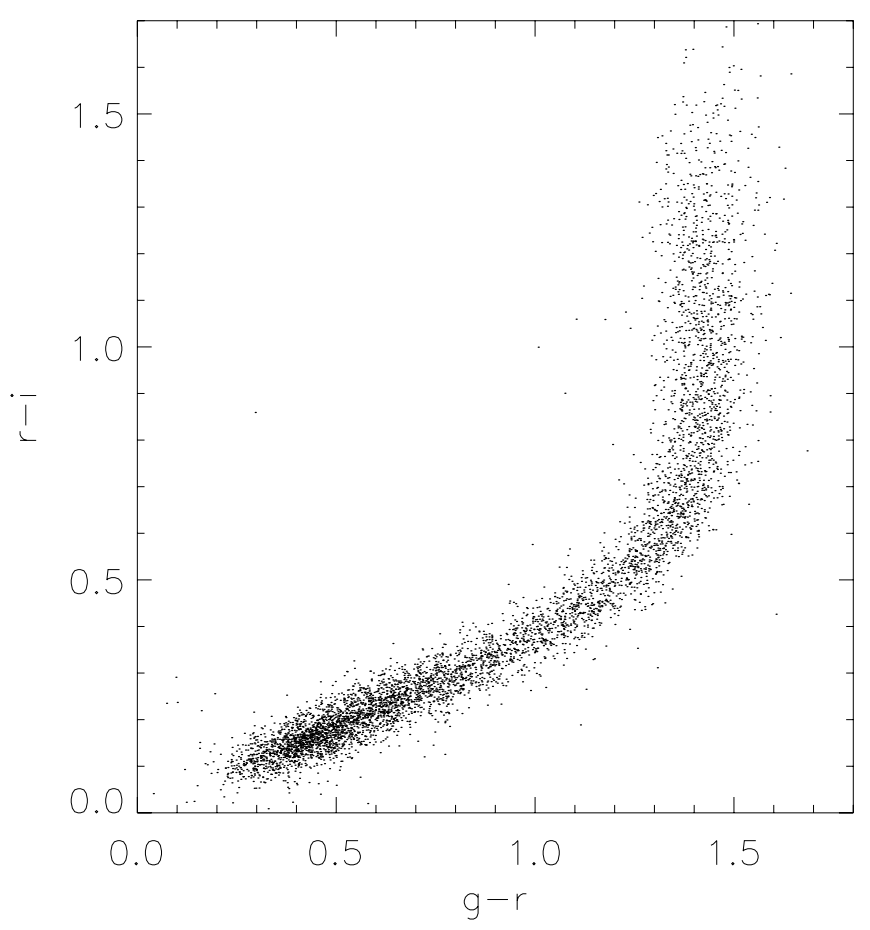

FIG. 8.-A $g-r-i$ color-color plot showing the stellar locus, based on the stars in 200 fields from camera column 3 of run 273, restricted to stars with apparent magnitude $r<19$. The colors are apparent colors based on PSF fluxes with no correction made for Galactic extinction. The IDL code that generated this figure is in $\S 4.2$.

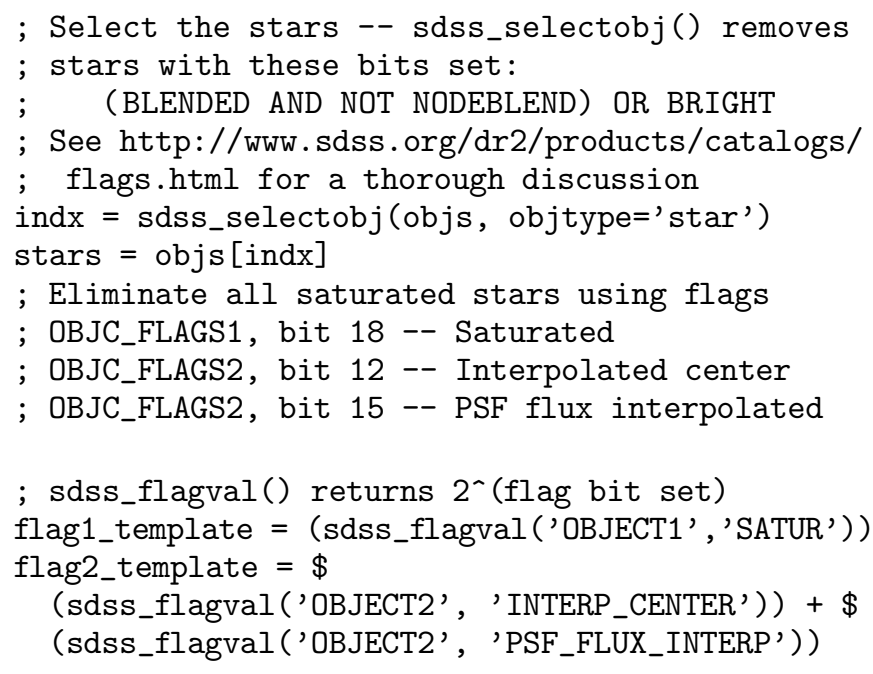

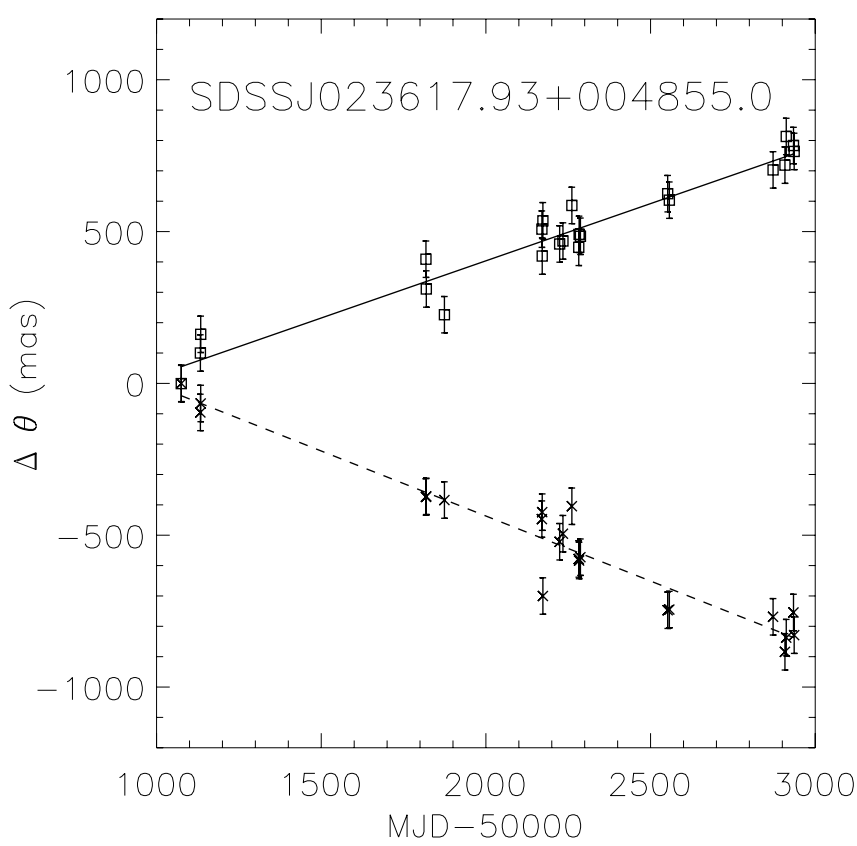

FIG. 9.-Relative position of the L6.5 brown dwarf SDSS J023617.93+ 004855.0 as a function of time. The solid line is a fit to the change in right ascension (relative to its fiducial position; squares), while the dashed line is a fit to the change in declination (crosses). The errors in the positions are 60 mas in both coordinates. The 22 SDSS observations clearly show the proper motion of this faint object (140 mas $\mathrm{yr}^{-1}$ in R.A., $-155 \mathrm{mas} \mathrm{yr}^{-1}$ in decl.). The code that generated this figure is in $\S 4.3$.

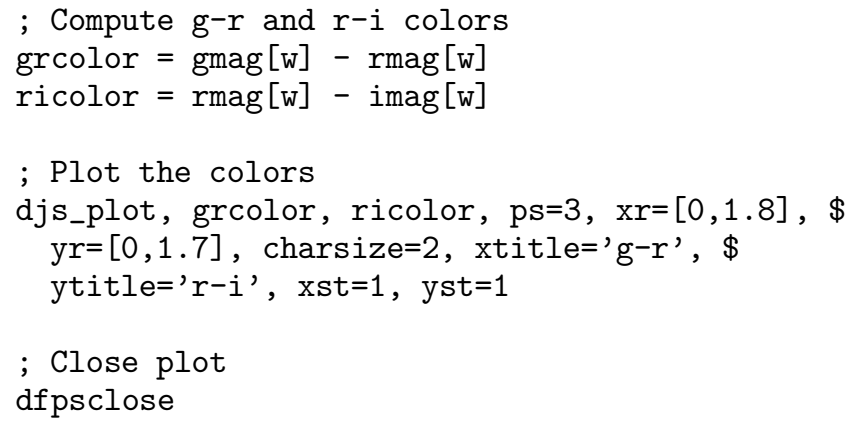

\subsection{The Proper Motion of a Brown Dwarf}

Multiple observations of the celestial equator enable timedomain studies. A particularly simple example is proper motions; Figure 9 shows the position of nearby L6.5 brown dwarf SDSS J023617.93+004855.0 (Schneider et al. 2002; Geballe et al. 2002) versus time. This object is in the SDSS southern survey equatorial region and has been observed multiple times, both during commissioning observations and during regular survey operations. The right ascension and declination offsets with respect to its fiducial position are shown versus time (MJD). The 22 observations span just over 5 years. The position is taken directly from the pipeline-processed data (for a discussion of SDSS astrometry, see Pier et al. 2003) and clearly show the proper motion of this faint object (140 mas $\mathrm{yr}^{-1}$ in right as-

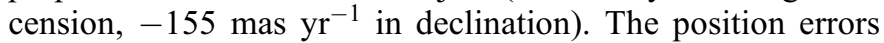
determined from this fit are 60 mas in each coordinate. Several SDSS brown dwarfs were identified in the commissioning and calibration data (Fan et al. 2000), and a project to measure the proper motions of nearby stars and substellar objects is under way. 
The following code fragment creates Figure 9; in particular, it demonstrates the use of sdss_findallobj to find multiple observations of objects in the data. Most observations of this object are not yet public; we include them in the figure to show what will be possible with the complete data set.

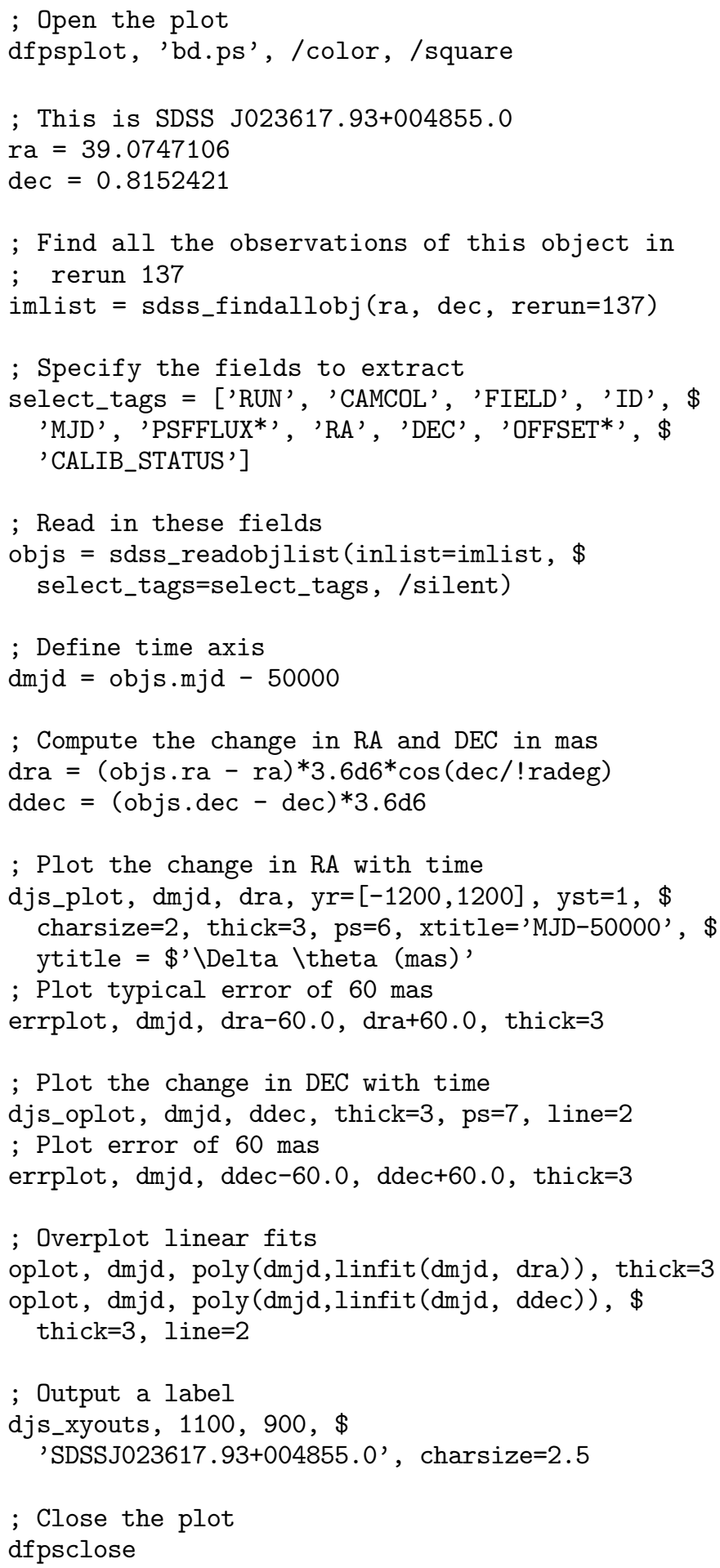

\subsection{Star Formation and Young Stellar Objects}

Figure 2 shows a color composite from the three most sensitive SDSS bands $(g, r$, and $i$ ) of a region from runs 259/273 centered on the star-forming region NGC 2068/NGC 2071/ HH 24-26. Note the line of Herbig-Haro objects in the outflow jet. Because their emission is dominated by emission lines,
Herbig-Haro objects have characteristic colors and can be automatically identified and counted in these regions of high obscuration. We find that there is a very different density distribution of these objects in the Taurus and Orion star-forming regions (Knapp et al. 2004).

The spectral energy distributions of the stars in NGC 2068 and NGC 2071 can be constructed from the five SDSS and three 2MASS bands, giving the luminosity function, the frequency of low-mass objects and the incidence of circumstellar dust. UV-excess objects, that is, young T Tauri stars, can be discovered and mapped across the Orion and Taurus starforming regions (McGehee et al. 2004b).

\subsection{Structure of the Galactic Halo and Vertical Structure of the Disk}

Because of the depth and color discrimination of SDSS photometry, the SDSS data have enabled major contributions to studies of the structure of the Galactic halo (Yanny et al. 2000; Ivezić et al. 2000; Odenkirchen et al. 2001; Newberg et al. 2003b). These and other studies of distant halo objects (Ibata et al. 1995; Johnston et al. 1995; Majewski et al. 2003; Martínez-Delgado et al. 2004) show the presence of long streams in the halo produced by the tidal disruption of satellite systems (small galaxies and loosely bound globular clusters). The data described herein allow these streams to be traced much closer to the Galactic plane. The SDSS stellar data at low Galactic latitudes can be used to investigate the relationships among the thin disk, the thick disk, and the Galactic halo, tracing the metallicity of each component. The discovery of these halo structures, and the ability of SDSS to follow the structure close to the Galactic plane, provide the impetus for a new initiative, known as SEGUE, to use the SDSS hardware and software to map the structure of the Galactic halo and the disk-halo interface (Newberg et al. 2003a; "Sloan Extension for Galactic Underpinnings and Evolution").

The vast majority of the red stars detected by SDSS are latetype disk dwarfs and subdwarfs. Hawley et al. (2002, 2004) demonstrate a well-established relationship between color and absolute magnitude for dwarfs of spectral type M0 and later, which allows the vertical mapping of the disk to a distance of about $1 \mathrm{kpc}$ from the Galactic plane as well as the threedimensional distribution of the interstellar dust. Measurements at different Galactic latitudes, especially at low latitudes, allow the measurement of the disk scale length and disk scale height for these stars (McGehee et al. 2004a, 2004b; Jurić et al. 2004).

\subsection{Properties of Interstellar Dust}

Reddening measured by fitting the position of the "blue tip" of the stellar locus in $(u, g, r, i, z)$ color space can be used to investigate possible deviations from the standard extinction law by comparing $E(u-g)$ with $E(g-r)$ in different directions (Finkbeiner et al. 2004). The mosaic images in Figures 2, 3, and 4 demonstrate that the dust clouds also have different emission or reflection properties. In particular, the Taurus dark clouds (Fig. 3) glow at red wavelengths, perhaps because of extended red emission (Witt et al. 1998). Differences between the emission properties of dust clouds, and of the illuminating starlight, can be investigated over large areas with these data.

Partial support for the computer systems required to process, store, and distribute these data was provided by NASA through grant NAG 5-6734, by NASA's Hubble Fellow program, and 
TABLE A1

calibobj BookKeEPING VALUes

\begin{tabular}{|c|c|c|}
\hline Name & Type & Description \\
\hline RUN ............................... & LONG & Run number \\
\hline RERUN ........................ & STRING & Rerun name \\
\hline CAMCOL ........................ & LONG & Camera column $(1-6)$ \\
\hline FIELD ............................ & LONG & Field number \\
\hline 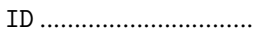 & LONG & Object ID, starting at 1 and unique in each field \\
\hline PARENT ...................... & LONG & ID of parent, -1 if this is a parent object \\
\hline NCHILD ........................ & LONG & Number of children deblended from this object \\
\hline MJD ............................... & LONG & Modified Julian Day for date of observation \\
\hline TAI .............................. & DOUBLE [5] & Mean time of observation for the object center in each filter, where TAI $=24 \times 3600 \times$ MJD \\
\hline AIRMASS .................... & FLOAT $[5]$ & Air mass approximated as csc (zenith angle) \\
\hline PSP_STATUS ................ & LONG [5] & "Status" field from the psField file \\
\hline CALIB_STATUS............ & INT [5] & Status of photometric calibration \\
\hline
\end{tabular}

by Princeton University. The NASA funds were originally provided in support of an Associate Investigator Program with WIRE. We thank NASA and the WIRE principal investigator, Perry Hacking, both for the opportunity to work in the WIRE team and for generously permitting us to apply the funding toward support of the Orion data reduction. D. P. F. is a Hubble Fellow supported by HST-HF-00129.01-A; C. R. is a Hubble Fellow supported by HST-HF-01143.01-A. We also thank Princeton University for generous support. This research made use of the IDL Astronomy User's Library at Goddard Space Flight Center. ${ }^{48}$

This publication makes use of data products from the Two Micron All Sky Survey, which is a joint project of the University of Massachusetts and the Infrared Processing and Analysis Center/California Institute of Technology, funded by the National Aeronautics and Space Administration and the National Science Foundation.

Funding for the SDSS has been provided by the Alfred P. Sloan Foundation, the Participating Institutions, the National Aeronautics and Space Administration, the National Science Foundation, the US Department of Energy, the Japanese Monbukagakusho, and the Max-Planck-Gesellschaft. The SDSS Web site is http://www.sdss.org/. SDSS is managed by the Astrophysical Research Consortium for the Participating Institutions. The Participating Institutions are the University of Chicago, Fermilab, the Institute for Advanced Study, the Japan Participation Group, Johns Hopkins University, Los Alamos National Laboratory, the Max-Planck-Institut für Astronomie,

\footnotetext{
${ }^{48}$ See http://idlastro.gsfc.nasa.gov/.
}

the Max-Planck-Institut für Astrophysik, New Mexico State University, the University of Pittsburgh, Princeton University, the US Naval Observatory, and the University of Washington.

\section{APPENDIX A}

\section{STRUCTURE OF THE CALIBOBJ FILES}

A current description of the calibObj data format is available at the Web site and is reproduced here in Tables A1-A3 for convenience. Fields unchanged from the $\mathrm{fpObjc}$ files are not repeated here and are described in Stoughton et al. (2002).

\section{APPENDIX B}

\section{USING INVERSE VARIANCE}

Flux errors are expressed as inverse variance ("ivar") in the calibObj files. This is convenient for inverse-variance weighting of combined quantities and handles zero signalto-noise ratio (ivar $=0$ ) gracefully, as the following example demonstrates.

\section{B1. COLOR CUT}

To select all stars with flux ratio $r / i$ greater than some color ratio $c$ with $n \sigma$ confidence, one writes the inequality as $r-$ $i c>n \sigma$, where $\sigma$ is the uncertainty in the quantity $r-i c$, or

$$
\sigma=\left(\frac{1}{I_{r}}+\frac{c^{2}}{I_{i}}\right)^{1 / 2}
$$

TABLE A2

calibobj Calibration Parameters

\begin{tabular}{|c|c|c|}
\hline Name & Type & Description \\
\hline NMGYPERCOUNT .................. & FLOAT [5] & Calibration (nanomaggies per count) \\
\hline NMGYPERCOUNT_IVAR ....... & FLOAT $[5]$ & $\begin{array}{l}\text { Formal error in the photometric calibration as an inverse variance; zero if there were no calibration stars and a } \\
\text { default calibration was used instead }\end{array}$ \\
\hline CLOUDCAM ........................... & INT [5] & Photometricity in each filter: $-1=$ unknown, $0=$ cloudy, $1=$ clear \\
\hline EXTINCTION..................... & FLOAT $[5]$ & $\begin{array}{l}\text { Galactic extinction in magnitudes, defined as }(5.155,3.793,2.751,2.086,1.479) E(B-V) \text {, where } E(B-V) \text { is from } \\
\text { the SFD } 98 \text { dust maps. Fluxes (and their errors) can be corrected for Galactic extinction as follows: } \\
\text { corrected flux }=\text { flux } \times 10^{\text {extinction } / 2.5}\end{array}$ \\
\hline PIXSCALE .......................... & FLOAT [5] & Pixel scale $\left(\operatorname{arcsec}\right.$ pixel $\left.^{-1}\right)$ \\
\hline 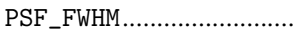 & FLOAT [5] & PSF FWHM (arcsec) \\
\hline PHI_OFFSET..................... & FLOAT [5] & Calibration angle (deg) to add to any uncalibrated position angles such that they mean degrees east of north \\
\hline
\end{tabular}


TABLE A3

calibobj Structure Calibrated Quantities

\begin{tabular}{|c|c|c|}
\hline Name & Type & Description \\
\hline $\mathrm{RA}$ & DOUBLE & J2000 right ascension (deg) in canonical ( $r$-band) filter \\
\hline 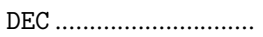 & DOUBLE & Declination (deg) in canonical ( $r$-band) filter \\
\hline OFFSETRA ................... & FLOAT [5] & Right ascension of center in this filter relative to RA (arcsec) \\
\hline OFFSETDEC.................. & FLOAT [5] & Declination of center in this filter relative to RA (arcsec) \\
\hline PHI_ISO_DEG............. & FLOAT [5] & Isophotal fit, angle east of north (deg) \\
\hline PHI_DEV_DEG............. & FLOAT [5] & De Vaucouleurs fit, angle east of north (deg) \\
\hline PHI_EXP_DEG.............. & FLOAT [5] & Exponential fit, angle east of north (deg) \\
\hline SKYFLUX ..................... & FLOAT [5] & Sky level (nMgy $\operatorname{arcsec}^{-2}$ ) \\
\hline SKYFLUX_IVAR........... & FLOAT [5] & \\
\hline PSFFLUX ....................... & FLOAT [5] & PSF flux (nMgy) \\
\hline PSFFLUX_IVAR........... & FLOAT [5] & \\
\hline FIBERFLUX ................. & FLOAT [5] & Fiber flux, $3^{\prime \prime}$ diameter (nMgy) \\
\hline FIBERFLUX_IVAR ....... & FLOAT [5] & \\
\hline MODELFLUX ................... & FLOAT [5] & Model flux (nMgy) \\
\hline MODELFLUX_IVAR ...... & FLOAT [5] & \\
\hline PETROFLUX .................. & FLOAT [5] & Petrosian flux (nMgy) \\
\hline PETROFLUX_IVAR ....... & FLOAT [5] & \\
\hline DEVFLUX ...................... & FLOAT [5] & De Vaucouleurs flux (nMgy) \\
\hline DEVFLUX_IVAR........... & FLOAT [5] & \\
\hline EXPFLUX & FLOAT [5] & Exponential flux (nMgy) \\
\hline $\begin{array}{l}\text { EXPFLUX_IVAR } \ldots \ldots \ldots \ldots \\
\text { APERFLUX } \ldots \ldots \ldots \ldots \ldots \ldots \ldots . . . . . . . . . .\end{array}$ & $\begin{array}{l}\text { FLOAT [5] } \\
\text { FLOAT [NAPER , 5] }\end{array}$ & 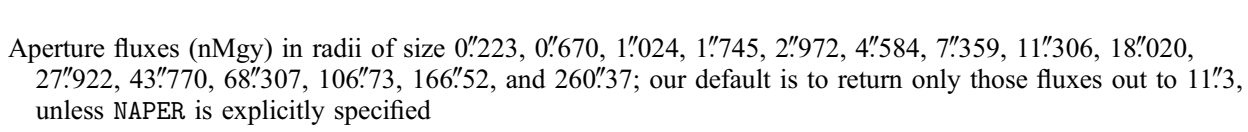 \\
\hline APERFLUX_IVAR ......... & FLOAT [NAPER , 5] & \\
\hline
\end{tabular}

where $I_{r}$ and $I_{i}$ are the $r$ - and $i$-band inverse variances, respectively. Multiplying by $\left(I_{r} I_{i}\right)^{1 / 2}$ yields the inequality

$$
(r-i c) \sqrt{I_{r} I_{i}}>n \sqrt{I_{i}+c^{2} I_{r}},
$$

which concisely expresses the desired condition without a division by any of the parameters and correctly handles the limiting cases of zero or negative flux, or inverse variance equal to zero (nonmeasurement). The conventional expression of this with magnitudes and $\sigma$ requires handling the special cases of zero or negative fluxes, or nonmeasurement, in either band.

Many astronomers will want to work with magnitudes, related to nanomaggy fluxes by

$$
m=22.5-2.5 \log \text { (flux), }
$$

but will find that any flux and color cuts are most easily performed in flux units. These fluxes and magnitudes are on the natural SDSS system and are offset from the true AB system by a few hundredths of a magnitude in each band (see Abazajian et al. 2004 for estimated offsets).

\section{B2. EXTINCTION CORRECTIONS}

Extinction values are presented in magnitudes for historical reasons. The extinction estimates provided are based on maps of IR dust emission (Schlegel et al. 1998) and are most reliable in the limit of long wavelengths. Extinction values are given in the calibObj files for each SDSS band, assuming a standard interstellar reddening law of $R_{V}=3.1$ in the parameterization of Cardelli et al. (1989). Because the SFD98 dust map is calibrated to $E(B-V)$, it is presented as $E(B-V)$ reddening. This has led to the misunderstanding that the presented $E(B-V)$ is valid for all values of $R_{V}$, when in fact it is the near-IR extinction (e.g., SDSS $z$ band) that is roughly constant with changes in $R_{V}$. Therefore, if the dust along a given line of sight is known to have a different value of $R_{V}$, it is necessary to extrapolate the extinction from a red band (e.g., $z$ band) to the desired band using the appropriate reddening law.

We remind the reader that flux uncertainties must be modified when correcting for extinction. Unlike magnitude uncertainties, which are fractional uncertainties, flux uncertainties are absolute and must be amplified by the same factor as the flux when extinction corrections are applied. To correct flux $F$ and inverse variance $I$ for an extinction of $A$ magnitudes, use

$$
F_{\text {corr }}=10^{0.4 A} F, \quad I_{\text {corr }}=10^{-0.8 A} I .
$$

Abazajian, K., et al. 2003, AJ, 126, 2081

$$
\text { 2004, AJ, 128, } 502
$$

Barth, A. J. 2001, in ASP Conf. Ser. 238, Astronomical Data Analysis Software and Systems X, ed. F. R. Harnden, Jr., F. A. Primini, \& H. E. Payne (San Francisco: ASP), 385

Becker, R. H., White, R. L., \& Helfand, D. J. 1995, ApJ, 450, 559

Cardelli, J. A., Clayton, G. C., \& Mathis, J. S. 1989, ApJ, 345, 245

Cutri, R. M., et al. 2003, Explanatory Supplement to the 2MASS All Sky Data Release (Pasadena: Caltech)

\section{REFERENCES}

Fan, X., et al. 2000, AJ, 119, 928

Finkbeiner, D. P., et al. 2004, in preparation

Fukugita, M., Ichikawa, T., Gunn, J. E., Doi, M., Shimasaku, K., \& Schneider, D. P. 1996, AJ, 111,1748

Geballe, T. R., et al. 2002, ApJ, 564, 466

Gunn, J. E., et al. 1998, AJ, 116, 3040

Hawley, S. L., et al. 2002, AJ, 123, 3409

. 2004, in preparation

Høg, E., et al. 2000, A\&A, 355, L27 (erratum 363, 385) 
Hogg, D. W., Finkbeiner, D. P., Schlegel, D. J., \& Gunn, J. E. 2001, AJ, 122, 2129

Ibata, R. A., Gilmore, G., \& Irwin, M. J. 1995, MNRAS, 277, 781

Ivezić, Ž., et al. 2000, AJ, 120, 963

Johnston, K. V., Spergel, D. N., \& Hernquist, L. 1995, ApJ, 451, 598

Jurić, M., et al. 2004, in preparation

Knapp, G. R., et al. 2004, in preparation

Kniazev, A. Yu., Grebel, E. K., Hao, L., Strauss, M. A., Brinkmann, J., \& Fukugita, M. 2003, ApJ, 593, L73

Lupton, R. H., Gunn, J. E., Ivezić, Ž., Knapp, G. R., Kent, S., \& Yasuda, N. 2001, in ASP Conf. Ser. 238, Astronomical Data Analysis Software and Systems X, ed. F. R. Harnden, Jr., F. A. Primini, \& H. E. Payne (San Francisco: ASP), 269

Lupton, R. H., Gunn, J. E., \& Szalay, A. S. 1999, AJ, 118, 1406

Lupton, R. H., Ivezić, Ž., Gunn, J. E., Knapp, G., Strauss, M. A., \& Yasuda, N. 2002, Proc. SPIE, 4836, 350

Lupton, R. H., et al. 2004, in preparation

Majewski, S., Skrutskie, M. F., Weinberg, M. D., \& Ostheimer, J. C. 2003, ApJ, 599,1082

Martínez-Delgado, D., Gómez-Flechoso, M. A., Aparacio, A., \& Carrera, R. 2004, ApJ, 601, 242

McGehee, P. M., Anderson, K. S. J., Hobbs, L. M., \& York, D. G. 2004a, in preparation
McGehee, P. M., et al. 2004b, in preparation

Monet, D.G., et al. 2003, AJ, 125, 984

Newberg, H. J., et al. 2003a, BAAS, 203, No. 112.11 . 2003b, ApJ, 596, L191

Odenkirchen, M., et al. 2001, ApJ, 548, L165

Oke, J. B., \& Gunn, J. E. 1983, ApJ, 266, 713

Pier, J. R., Munn, J. A., Hindsley, R. B., Hennessy, G. S., Kent, S. M., Lupton,

R. H., \& Ivezić, Ž. 2003, AJ, 125, 1559

Schlegel, D. J., Finkbeiner, D. P., \& Davis, M. 1998, ApJ, 500, 525 (SFD98)

Schlegel, D. J., et al. 2004, in preparation

Schneider, D. P., et al. 2002, AJ, 123, 458

Smith, J. A., et al. 2002, AJ, 123, 2121

Stoughton, C., et al. 2002, AJ, 123, 485

Witt, A. N., Gordon, K. D., \& Furton, D. G. 1998, ApJ, 501, L111

Yanny, B., et al. 2000, ApJ, 540, 825

York, D. G., et al. 2000, AJ, 120, 1579

Zacharias, N., Rafferty, T. J., \& Zacharias, M. I. 2000, in ASP Conf. Ser. 216, Astronomical Data Analysis Software and Systems IX, ed. N. Manset, C. Veillet, \& D. Crabtree (San Francisco: ASP), 427 